

\title{
Cooperative Network Coding Strategies for Wireless Relay Networks with Backhaul
}

IEEE Transactions on Communications, vol. 59, pp. 2502-2514, Sep. 2011.

(C) 2011 IEEE. Personal use of this material is permitted. However, permission to reprint/republish this material for advertising or promotional purposes or for creating new collective works for resale or redistribution to servers or lists, or to reuse any copyrighted component of this work in other works must be obtained from the IEEE.

JINFENG DU, MING XIAO, MIKAEL SKOGLUND

Stockholm September 2011

School of Electrical Engineering and the ACCESS Linnaeus Center, Royal Institute of Technology (KTH), SE-100 44 Stockholm, Sweden IR-EE-KT 2011:024 


\title{
Cooperative Network Coding Strategies for Wireless Relay Networks with Backhaul
}

\author{
Jinfeng Du, Student Member, IEEE, Ming Xiao, Member, IEEE, and Mikael Skoglund, Senior Member, IEEE
}

\begin{abstract}
We investigate cooperative network coding strategies for relay-aided two-source two-destination wireless networks with a backhaul connection between the source nodes. Each source multicasts information to all destinations using a shared relay. We study cooperative strategies based on different network coding schemes, namely, finite field and linear network coding, and lattice coding. To further exploit the backhaul connection, we also propose network coding based beamforming. We measure the performance in term of achievable rates over Gaussian channels, and observe significant gains over benchmark schemes. We derive the achievable rate regions for these schemes and find the cutset bound for our system. We also show that the cut-set bound can be achieved by network coding based beamforming when the signal-to-noise ratios lie in the sphere defined by the source-relay and relay-destination channel gains.
\end{abstract}

\section{INTRODUCTION}

Capacity bounds and various cooperative strategies for three-node relaying networks (source-relay-sink, or two cooperative sources and one sink) have been studied in [1], [2]. The relay (or the other source) uses decode-and-forward (DF) or compress-and-forward (CF) to aid the transmission. Coding schemes have been investigated for multiple-access relay channels (MARC) [3], [4] involving multiple sources and a single destination, and for broadcast relay channels (BRC) [3], [5] where a single source transmits messages to multiple destinations. Recent results on capacity bounds for multiple-source multiple-destination relay networks, [6]-[9] and references therein, have provided valuable insight into the benefits of relaying. Motivated by the MAC channel at the relay node where different messages mix up by nature, various network coding (NC) [10]-[12] approaches, which essentially combine multiple messages together, can be introduced to boost the sum rate. For instance, in a relay-aided two-source two-sink multicast network, achievable rates for a full-duplex amplify-and-forward (AF) relay with linear NC (LNC) have been studied in [13], and in [8] the relay uses lattice codes for network coding. In [14] joint $\mathrm{NC}$ and physical layer coding is performed via lattice coding for the bi-directional relay channel. The recently proposed noisy network coding scheme (Noisy NC) [15] for transmitting multiple sources over a general noisy network, has been shown to outperform

Manuscript received August 28, 2010; revised February 18, 2011.

This work was presented in part at IEEE ITW, Aug. 2010.

This work was supported in part by the Swedish Governmental Agency for Innovation Systems (VINNOVA) and the Swedish Foundation for Strategic Research (SSF).

Jinfeng Du, Ming Xiao and Mikael Skoglund are with School of Electrical Engineering and the ACCESS Linnaeus Center, Royal Institute of Technology, Stockholm, Sweden (Email: jinfeng@kth.se; ming.xiao@ee.kth.se; mikael.skoglund@ee.kth.se).

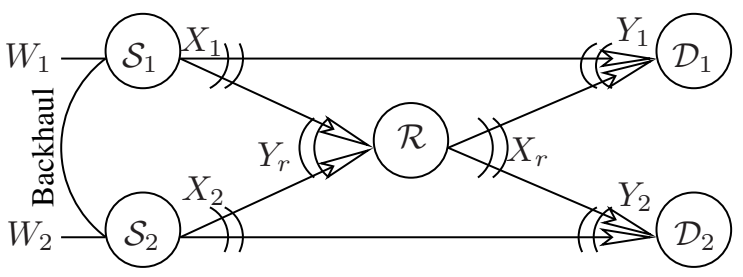

Figure 1. Two source nodes $\mathcal{S}_{1}$ and $\mathcal{S}_{2}$, connected with backhaul, multicast information $W_{1}$ and $W_{2}$ respectively to both destinations $\mathcal{D}_{1}$ and $\mathcal{D}_{2}$, with aid from a full-duplex relay node $\mathcal{R}$.

the conventional $\mathrm{CF}$ scheme in the Gaussian two-way relay channel and the interference relay channel. Apart from introducing dedicated relay nodes to help the transmission, one can also utilize cooperative strategies among sources [16][21] and/or among destinations [20]-[22] with the help of orthogonal conferencing channels.

In this paper, we aim at evaluating achievable rate regions for various cooperative strategies when source cooperation and network coding are designed jointly with the relaying. More specifically, we focus on a relay-aided two-source twodestination multicast network with backhaul support, as shown in Fig. 1. Sources $\mathcal{S}_{1}$ and $\mathcal{S}_{2}$ multicast their own information ( $W_{1}$ and $W_{2}$ respectively) to geographically separated destinations $\mathcal{D}_{1}$ and $\mathcal{D}_{2}$, with the help of a relay $\mathcal{R}$. This model arises, for example, in a wireless cellular downlink where two base stations multicast to two mobile terminals, one in each cell, with the help of a dedicated relay deployed at the common cell boundary. Since the base stations are connected through the (fiber or microwave) backhaul, more general network coding schemes can be used at the relay to cooperate with the sources' transmission. This model is interesting since it is a combination of relaying, MARC, BRC, source cooperation, and network coding. It can be extended to more general networks by tuning the channel gains within the range $[0, \infty)$. In this paper, we are interested in the scenario without cross channels between $\mathcal{S}_{1}$ and $\mathcal{D}_{2}$, or $\mathcal{S}_{2}$ and $\mathcal{D}_{1}$. While, in general, the signal from $\mathcal{S}_{i}$ would be heard also at $\mathcal{D}_{j}, j \neq i$, our assumption can be motivated for example in scenarios where the cross links are too weak to be of any use, or are technically suppressed. In any case we consider any contribution directly from $\mathcal{S}_{i}$ at $\mathcal{D}_{j}(j \neq i)$ not to be useful and therefore part of the noise. We also restrict our analysis to fixed channel gains, and we assume a full-duplex DF relay. Furthermore, any extensions of the cooperative NC strategies developed in this paper to multiple sources and/or multiple relays are left to future work. 
The paper is organized as follows. The system model is introduced in Sec. II. For symmetric channel gains and high-rate backhaul, various cooperative NC strategies are investigated in Sec. III, and a benchmark scheme together with the cutset bound are presented in Sec. IV. Cooperative NC strategies for non-symmetric channel gains and for low-rate backhaul (i.e., partial transmitter cooperation) scenarios are discussed in Sec. V. Numerical results are presented in Sec. VI and concluding remarks in Sec. VII.

Notation: Capital letter $X$ indicates a real valued random variable and $p(X)$ indicates its probability density/mass function. $X^{(n)}$ denotes a vector of random variables of length $n$, and with the $k$ th component $X[k]$ (in general without emphasizing the $\left.(\cdot)^{(n)}\right) . I(X ; Y)$ denotes the mutual information between $X$ and $Y$, and $C(x)=\frac{1}{2} \log _{2}(1+x)$ is the Gaussian capacity function.

\section{SySTEM MODEL}

To simplify our analysis, we first consider the symmetric channel gain scenario illustrated in Fig. 1

$$
\begin{aligned}
& Y_{1}^{(n)}=X_{1}^{(n)}+b X_{r}^{(n)}+Z_{1}^{(n)}, \\
& Y_{2}^{(n)}=X_{2}^{(n)}+b X_{r}^{(n)}+Z_{2}^{(n)}, \\
& Y_{r}^{(n)}=a X_{1}^{(n)}+a X_{2}^{(n)}+Z_{r}^{(n)},
\end{aligned}
$$

where $a \geq 0$ is the normalized channel gain for the sourcerelay links and $b \geq 0$ for the relay-destination links. For $i=1,2, r, X_{i}^{(n)}, Y_{i}^{(n)}$ and $Z_{i}^{(n)}$ are $n$-dimensional transmitted signals, received signals, and noise, respectively, where $Z_{i}[k]$, $k=1, \ldots, n$ are i.i.d. Gaussian with zero-mean and unitvariance. The transmitted signals are subject to individual average power constraints, i.e.,

$$
\frac{1}{n} \sum_{k=1}^{n} X_{i}^{2}[k] \leq P_{i}, \quad i=1,2, r .
$$

Note that (1) implies simultaneously perfect synchronization at $\mathcal{D}_{1}, \mathcal{D}_{2}$, and $\mathcal{R}$, respectively. This assumption, although widely adopted in information-theoretic work, is optimistic in practice. In general, the results we obtain based on perfect synchronization will serve as upper bounds on any practical performance, and can be directly extended in the same way as in [2] to scenarios where constructive (co-phase) addition is not available.

In practice the backhaul normally has much higher capacity and lower error rates than the forward wireless channels. Therefore, in our model the backhaul is assumed to be error-free and of sufficiently high capacity (higher than the forward sum-rate). The case of a backhaul capacity smaller than the sum-rate will be discussed in Sec. V. With a high rate backhaul, our system is closely related to the MIMO relay channel scenario, as studied in [23], [24]. However the problems are not equivalent, and we emphasize the following three main differences between the system investigated in this paper and the MIMO relay scenario with a two-antenna source node. First, in our system each source/antenna is subject to an individual power constraint (2), while in the MIMO relay channel model a sum-power constraint is usually applied at the source node, which in general implies a larger achievable rate region. Second, in our system the relay combines the messages from the sources by performing NC rather than forwarding them separately through orthogonal channels. Last but not the least, the cooperative strategies proposed for high rate backhaul in Sec. III can be directly extended to the finiterate backhaul scenario with the help of superposition coding or time-sharing strategies, as stated in Sec. V.

\section{COOPERATIVE NETWORK CODING STRATEGIES}

Similar to [1]-[3], [6], source $\mathcal{S}_{i}, i=1,2$, divides its messages $W_{i}$ into $B$ blocks $W_{i, 1}, \ldots, W_{i, B}$ with $n R_{i}$ bits each. The transmission is completed over $B+1$ blocks. At the first block the two sources exchange $W_{i, 1}$ over the backhaul and also broadcast their own messages over the relay channels; in block $t$, source $\mathcal{S}_{i}$ exchanges $W_{i, t}$ through the backhaul and broadcasts its codeword $X_{i, t}^{(n)}$, which is a function of $\left(W_{i, t}, W_{1, t-1}, W_{2, t-1}\right)$, over the channels; in block $B+1$ only $W_{i, B}$ is broadcasted. As each transmission is over $n$ channel uses, and assuming the backhaul is used for free, the overall rate is $\frac{B n R_{i}}{(B+1) n}$ bits per channel use, which converges to $R_{i}$ when $B$ goes to infinity. Three decoding protocols, namely successive decoding [1], backward decoding [25], and sliding-window decoding [26], have been summarized and extended to multiple-source or multiple-relay scenarios in [3]. We implement these protocols at relay/destination nodes depending on the cooperative NC strategy under consideration. Unless stated otherwise, random coding is used for encoding and joint-typicality is used for decoding. Each codeword is generated randomly in the memoryless fashion [27]: For transmitting messages in $\{W\}$ each of $n R$ bits, we create a codebook consisting of $2^{n R}$ randomly and independently generated sequences $\left\{U^{(n)}\right\}$, each of $n$-bit length, according to the distribution $\Pi_{i=1}^{n} p\left(u_{i}\right)$. We assign a codeword $U^{(n)}$ to a message $W$ and associate them via an encoding function $U^{(n)}(W)$, omitting the explicit relation where appropriate.

\section{A. Finite-field Network Coding with DF $(D F+F N C)$}

At the end of block $t-1$, the relay decodes $\left(W_{1, t-1}, W_{2, t-1}\right)$ jointly from its received signal $Y_{r, t-1}^{(n)}$ and then creates a new message $W_{r, t}=W_{1, t-1} \oplus W_{2, t-1}$ (bitwise $\mathrm{GF}(2)$ addition). If the lengths of $W_{1, t-1}$ and $W_{2, t-1}$ are not equal, i.e., $R_{1} \neq R_{2}$, we can append zeros at the end of the shorter message. During block $t, \mathcal{R}$ transmits $W_{r, t}$ using an independent random codebook $\left\{U^{(n)}\right\}$ of size $2^{n R}$ (where $R=\max \left(R_{1}, R_{2}\right)$,

$$
X_{r, t}^{(n)}=\sqrt{P_{r}} U^{(n)}\left(W_{r, t}\right) .
$$

$\mathcal{S}_{1}$ and $\mathcal{S}_{2}$, on the other hand, transmit their information via independent random codebooks $\left\{V_{1}^{(n)}\right\}$ of size $2^{n R_{1}}$ and $\left\{V_{2}^{(n)}\right\}$ of size $2^{n R_{2}}$, respectively. Since $W_{1, t-1}$ and $W_{2, t-1}$ are exchanged via the backhaul in block $t-1, \mathcal{S}_{1}$ and $\mathcal{S}_{2}$ also know $W_{r, t}$ if decoding at $\mathcal{R}$ is reliable. Therefore to exploit the possibility of coherent combining gain, $\mathcal{S}_{1}$ and $\mathcal{S}_{2}$ can coordinate their transmission with $\mathcal{R}$ as follows,

$$
\begin{aligned}
X_{1, t}^{(n)} & =\sqrt{\alpha_{1} P_{1}} V_{1}^{(n)}\left(W_{1, t}\right)+\sqrt{\left(1-\alpha_{1}\right) P_{1}} U^{(n)}\left(W_{r, t}\right), \\
X_{2, t}^{(n)} & =\sqrt{\alpha_{2} P_{2}} V_{2}^{(n)}\left(W_{2, t}\right)+\sqrt{\left(1-\alpha_{2}\right) P_{2}} U^{(n)}\left(W_{r, t}\right),
\end{aligned}
$$


Table I

ILLUSTRATION OF THE ENCODING AND DECODING PROCESS FOR DF+FNC, WITH $W_{r, t}=W_{1, t-1} \oplus W_{2, t-1}, W_{r, 1}=1$, AND $B=3$.

\begin{tabular}{|c|c|c|c|c|}
\hline$t=$ & 1 & 2 & 3 & 4 \\
\hline$\rightleftharpoons$ & $W_{1,1} \Leftrightarrow W_{2,1}$ & $W_{1,2} \Leftrightarrow W_{2,2}$ & $W_{1,3} \Leftrightarrow W_{2,3}$ & $/$ \\
\hline $\mathcal{S}_{1}$ transmits & $\left(W_{1,1}, 1\right)$ & $\left(W_{1,2}, W_{r, 2}\right)$ & $\left(W_{1,3}, W_{r, 3}\right)$ & $\left(1, W_{r, 4}\right)$ \\
\hline $\mathcal{S}_{2}$ transmits & $\left(W_{2,1}, 1\right)$ & $\left(W_{2,2}, W_{r, 2}\right)$ & $\left(W_{2,3}, W_{r, 3}\right)$ & $\left(1, W_{r, 4}\right)$ \\
\hline $\mathcal{R}$ transmits & 1 & $W_{r, 2}$ & $W_{r, 3}$ & $W_{r, 4}$ \\
\hline $\mathcal{R}$ decodes & $W_{1,1}, W_{2,1}$ & $W_{1,2}, W_{2,2}$ & $W_{1,3}, W_{2,3}$ & $/$ \\
\hline $\mathcal{D}_{1}$ decodes & $W_{1,1}$ & $W_{1,2}, W_{r, 2}$ & $W_{1,3}, W_{r, 3}$ & $W_{r, 4}$ \\
recovers by $\oplus$ & $/$ & $W_{2,1}$ & $W_{2,2}$ & $W_{2,3}$ \\
\hline
\end{tabular}

where $0 \leq \alpha_{1}, \alpha_{2} \leq 1$ are power allocation parameters. The received signals are therefore

$$
\begin{array}{r}
Y_{1, t}^{(n)}=\sqrt{\alpha_{1} P_{1}} V_{1}^{(n)}+\left(\sqrt{\left(1-\alpha_{1}\right) P_{1}}+b \sqrt{P_{r}}\right) U^{(n)}+Z_{1, t}^{(n)}, \\
Y_{2, t}^{(n)}=\sqrt{\alpha_{2} P_{2}} V_{2}^{(n)}+\left(\sqrt{\left(1-\alpha_{2}\right) P_{2}}+b \sqrt{P_{r}}\right) U^{(n)}+Z_{2, t}^{(n)}, \\
Y_{r, t}^{(n)}=a\left(\sqrt{\left(1-\alpha_{1}\right) P_{1}}+\sqrt{\left(1-\alpha_{2}\right) P_{2}}\right) U^{(n)}+a \sqrt{\alpha_{1} P_{1}} V_{1}^{(n)} \\
+a \sqrt{\alpha_{2} P_{2}} V_{2}^{(n)}+Z_{r, t}^{(n)} .
\end{array}
$$

Successive decoding is implemented at both the relay and the two destination nodes: assuming $W_{1, t-1}$ has been successfully decoded by $\mathcal{D}_{1}$, at the end of block $t, \mathcal{D}_{1}$ recovers $\left(W_{1, t}, W_{r, t}\right)$ jointly from $Y_{1, t}^{(n)}$, and then retrieves $W_{2, t-1}=$ $W_{r, t} \oplus W_{1, t-1}$. This approach is also used for $\mathcal{D}_{2}$. The relay $\mathcal{R}$ decodes jointly $\left(W_{1, t}, W_{2, t}\right)$ from $Y_{r, t}^{(n)}$ by first cancelling out $U^{(n)}$. The encoding/decoding process is illustrated in Table I.

Proposition 1: The achievable rate region for $\mathrm{DF}+\mathrm{FNC}$ is the union over all $\left(R_{1}, R_{2}\right)$ satisfying

$R_{1}<\min \left\{C\left(a^{2} \alpha_{1} P_{1}\right), C\left(\alpha_{1} P_{1}\right), C\left(\left(\sqrt{\left(1-\alpha_{2}\right) P_{2}}+b \sqrt{P_{r}}\right)^{2}\right)\right\}$,

$R_{2}<\min \left\{C\left(a^{2} \alpha_{2} P_{2}\right), C\left(\alpha_{2} P_{2}\right), C\left(\left(\sqrt{\left(1-\alpha_{1}\right) P_{1}}+b \sqrt{P_{r}}\right)^{2}\right)\right\}$,

$R_{1}+R_{2}<\min \left\{C\left(P_{1}+b^{2} P_{r}+2 b \sqrt{\left(1-\alpha_{1}\right) P_{1} P_{r}}\right)\right.$,

$$
\left.C\left(a^{2} \alpha_{1} P_{1}+a^{2} \alpha_{2} P_{2}\right), C\left(P_{2}+b^{2} P_{r}+2 b \sqrt{\left(1-\alpha_{2}\right) P_{2} P_{r}}\right)\right\},
$$

where the union is taken over $0 \leq \alpha_{1}, \alpha_{2} \leq 1$.

Proof: The proof can be found in Appendix A.

The constraint on $R_{1}$ corresponds to the condition that $W_{1}$ can be decoded reliably at $\mathcal{R}$ and $\mathcal{D}_{1}$, and that the NC message $W_{r}$ can be decoded at $\mathcal{D}_{2}$, and similarly for $R_{2}$ and $R_{1}+R_{2}$. Note that our scheme is similar to the strategy in [8]: $\mathcal{D}_{1}$ recovers $W_{1}$ from the direct link and $W_{r}$ from the $\mathcal{R}-\mathcal{D}_{1}$ link, and then retrieves $W_{2}$ based on the observation of $W_{1}$ and $W_{r}$. But there are two main differences: finite-field NC rather than lattice coding is used; both source nodes know $W_{r}$ thanks to the backhaul and therefore they cooperate with $\mathcal{R}$ to get a coherent combining gain.

Corollary 1: For the symmetric scenario with $P_{1}=P_{2}=$ $P_{r}=P$ and $R_{1}=R_{2}=R$, rate $R$ is achievable by $\mathrm{DF}+\mathrm{FNC}$ if

$R<\max _{0 \leq \alpha \leq 1} \min \left\{C(\alpha P), \frac{C\left(2 a^{2} P \alpha\right)}{2}, \frac{C\left(\left(1+b^{2}+2 b \sqrt{1-\alpha}\right) P\right)}{2}\right\}$

Proof: The result follows straightforwardly from (6) by setting $\alpha_{1}=\alpha_{2}=\alpha$.

Without the backhaul, $\mathcal{S}_{1}$ and $\mathcal{S}_{2}$ cannot know/estimate $W_{r}$ and therefore cannot cooperate with $\mathcal{R}$, i.e. $\alpha_{1}=\alpha_{2}=1$. Hence, no coherent combining gain can be achieved.

\section{B. Linear Network Coding with $D F(D F+L N C)$}

When LNC is used in the signal domain, $\mathcal{R}$ essentially performs superposition coding. The scheme presented here is a natural extension of the one in Theorem 1 of [6] which is designed for transmitting both private and common messages via the interference relay channel (IFRC). In our case, only common messages are transmitted (i.e., multicast). Unlike in [6] where each source can only cooperate with node $\mathcal{R}$ regarding its own message in $X_{r}^{(n)}$, the two source nodes can in our case cooperate to transmit both messages, thanks to the backhaul. We first generate two independent random codebooks $\left\{U_{1}^{(n)}\right\}$ of size $2^{n R_{1}}$ and $\left\{U_{2}^{(n)}\right\}$ of size $2^{n R_{2}}$. At the end of block $t-1, \mathcal{R}$ decodes $\left(W_{1, t-1}, W_{2, t-1}\right)$ and then picks up codewords $U_{1}^{(n)}\left(W_{1, t-1}\right)$ and $U_{2}^{(n)}\left(W_{2, t-1}\right)$ from the two codebooks respectively, and transmits the superposition of these in block $t$ with power allocation parameter $0 \leq \alpha_{r} \leq 1$

$X_{r, t}^{(n)}=\sqrt{\alpha_{r} P_{r}} U_{1}^{(n)}\left(W_{1, t-1}\right)+\sqrt{\left(1-\alpha_{r}\right) P_{r}} U_{2}^{(n)}\left(W_{2, t-1}\right)$.

For each codeword $U_{1}^{(n)}\left(W_{1, t-1}\right)$, we generate an independent codebook $\left\{V_{1}^{(n)}\right\}$ of size $2^{n R_{1}}$, and then use this codebook to encode the new message $W_{1, t}$. We denote the selected codeword for $W_{1, t}$ given $W_{1, t-1}$ as $V_{1}^{(n)}\left(W_{1, t}, W_{1, t-1}\right)$. Similarly we choose $V_{2}^{(n)}\left(W_{2, t}, W_{2, t-1}\right)$ for $W_{2, t}$. With power allocation parameters $0 \leq \alpha_{i}^{\prime}, \alpha_{i}^{\prime \prime} \leq 1, i=1,2$ to cooperate with $\mathcal{R}$, the transmitted signal at $\mathcal{S}_{1}$ and $\mathcal{S}_{2}$ are therefore

$X_{1, t}^{(n)}=\sqrt{\alpha_{1}^{\prime} P_{1}} U_{1}^{(n)}+\sqrt{\alpha_{1}^{\prime \prime} P_{1}} U_{2}^{(n)}+\sqrt{\left(1-\alpha_{1}^{\prime}-\alpha_{1}^{\prime \prime}\right) P_{1}} V_{1}^{(n)}$
$X_{2, t}^{(n)}=\sqrt{\alpha_{2}^{\prime} P_{2}} U_{2}^{(n)}+\sqrt{\alpha_{2}^{\prime \prime} P_{2}} U_{1}^{(n)}+\sqrt{\left(1-\alpha_{2}^{\prime}-\alpha_{2}^{\prime \prime}\right) P_{2}} V_{2}^{(n)}$.

The received signals at the destinations and the relay are

$$
\begin{array}{r}
Y_{1}^{(n)}=\sqrt{\left(1-\alpha_{1}^{\prime}-\alpha_{1}^{\prime \prime}\right) P_{1}} V_{1}^{(n)}+\left(\sqrt{\alpha_{1}^{\prime} P_{1}}+b \sqrt{\alpha_{r} P_{r}}\right) U_{1}^{(n)} \\
+\left(\sqrt{\alpha_{1}^{\prime \prime} P_{1}}+b \sqrt{\left(1-\alpha_{r}\right) P_{r}}\right) U_{2}^{(n)}+Z_{1}^{(n)}, \\
Y_{2}^{(n)}=\sqrt{\left(1-\alpha_{2}^{\prime}-\alpha_{2}^{\prime \prime}\right) P_{2}} V_{2}^{(n)}+\left(\sqrt{\alpha_{2}^{\prime \prime} P_{2}}+b \sqrt{\alpha_{r} P_{r}}\right) U_{1}^{(n)} \\
+\left(\sqrt{\alpha_{2}^{\prime} P_{2}}+b \sqrt{\left(1-\alpha_{r}\right) P_{r}}\right) U_{2}^{(n)}+Z_{2}^{(n)}, \\
Y_{r}^{(n)}=a\left[\sqrt{\left(1-\alpha_{1}^{\prime}-\alpha_{1}^{\prime \prime}\right) P_{1}} V_{1}^{(n)}+\sqrt{\left(1-\alpha_{2}^{\prime}-\alpha_{2}^{\prime \prime}\right) P_{2}} V_{2}^{(n)}\right. \\
\left.+\left(\sqrt{\alpha_{1}^{\prime} P_{1}}+\sqrt{\alpha_{2}^{\prime \prime} P_{2}}\right) U_{1}^{(n)}+\left(\sqrt{\alpha_{1}^{\prime \prime} P_{1}}+\sqrt{\alpha_{2}^{\prime} P_{2}}\right) U_{2}^{(n)}\right]+Z_{r}^{(n)} .
\end{array}
$$

The decoding follows directly from [6]: the relay performs successive decoding and the destinations use backward decoding. $\mathcal{R}$ decodes $\left(W_{1, t}, W_{2, t}\right)$ reliably from $Y_{r, t}^{(n)}$ at the end of block $t$. $\mathcal{D}_{1}$ and $\mathcal{D}_{2}$ start decoding when transmission is finished. At block $B+1$, no new message is transmitted and the received signal at $\mathcal{D}_{1}\left(\mathcal{D}_{2}\right)$ only depends on $\left(W_{1, B}, W_{2, B}\right)$. After decoding $\left(W_{1, B}, W_{2, B}\right)$ successfully, only $W_{1, B-1}$ ( $\left.W_{2, B-1}\right)$ is unknown in $Y_{1, B}^{(n)}\left(Y_{2, B}^{(n)}\right)$, and we repeat this process backwards until all messages are recovered.

Proposition 2: The achievable rate region for $\mathrm{DF}+\mathrm{LNC}$ is 
given by

$$
\begin{gathered}
R_{1}<\min \left\{C\left(a^{2} P_{1}\left(1-\alpha_{1}^{\prime}-\alpha_{1}^{\prime \prime}\right)\right),\right. \\
C\left(\left(1-\alpha_{1}^{\prime \prime}\right) P_{1}+b^{2} \alpha_{r} P_{r}+2 b \sqrt{\alpha_{1}^{\prime} \alpha_{r} P_{1} P_{r}}\right), \\
\left.C\left(\alpha_{2}^{\prime \prime} P_{2}+b^{2} \alpha_{r} P_{r}+2 b \sqrt{\alpha_{2}^{\prime \prime} \alpha_{r} P_{2} P_{r}}\right)\right\}, \\
R_{2}<\min \left\{C\left(a^{2} P_{2}\left(1-\alpha_{2}^{\prime}-\alpha_{2}^{\prime \prime}\right)\right),\right. \\
C\left(\left(1-\alpha_{2}^{\prime \prime}\right) P_{2}+b^{2}\left(1-\alpha_{r}\right) P_{r}+2 b \sqrt{\alpha_{2}^{\prime}\left(1-\alpha_{r}\right) P_{2} P_{r}}\right), \\
\left.C\left(\alpha_{1}^{\prime \prime} P_{1}+b^{2}\left(1-\alpha_{r}\right) P_{r}+2 b \sqrt{\alpha_{1}^{\prime \prime}\left(1-\alpha_{r}\right) P_{1} P_{r}}\right)\right\}, \\
R_{1}+R_{2}<\min \left\{C\left(a^{2}\left(1-\alpha_{1}^{\prime}-\alpha_{1}^{\prime \prime}\right) P_{1}+a^{2}\left(1-\alpha_{2}^{\prime}-\alpha_{2}^{\prime \prime}\right) P_{2}\right),\right. \\
C\left(P_{1}+b^{2} P_{r}+2 b \sqrt{P_{1} P_{r}}\left[\sqrt{\alpha_{1}^{\prime} \alpha_{r}}+\sqrt{\alpha_{1}^{\prime \prime}\left(1-\alpha_{r}\right)}\right]\right), \\
\left.C\left(P_{2}+b^{2} P_{r}+2 b \sqrt{P_{2} P_{r}}\left[\sqrt{\alpha_{2}^{\prime \prime} \alpha_{r}}+\sqrt{\alpha_{2}^{\prime}\left(1-\alpha_{r}\right)}\right]\right)\right\},
\end{gathered}
$$

with the union taken over all $0 \leq \alpha_{r}, \alpha_{1}^{\prime}, \alpha_{1}^{\prime \prime}, \alpha_{2}^{\prime}, \alpha_{2}^{\prime \prime} \leq 1$, with $\alpha_{1}^{\prime}+\alpha_{1}^{\prime \prime} \leq 1, \alpha_{2}^{\prime}+\alpha_{2}^{\prime \prime} \leq 1$.

Proof: The proof can be found in Appendix B.

The constraint on $R_{1}$ refers to the condition that $W_{1}$ can be decoded successfully at $\mathcal{R}, \mathcal{D}_{1}$, and $\mathcal{D}_{2}$, respectively, and similarly for $R_{2}$ and $R_{1}+R_{2}$.

Corollary 2: For the symmetric scenario, the following equal rate constraints apply

$$
\begin{aligned}
R<\max _{\substack{\alpha^{\prime} \geq 0, \alpha^{\prime \prime} \geq 0 \\
0 \leq \alpha^{\prime}+\alpha^{\prime \prime} \leq 1}} \min \left\{C\left(\left(\alpha^{\prime \prime}+\frac{1}{2} b^{2}+b \sqrt{2 \alpha^{\prime \prime}}\right) P\right),\right. \\
C\left(\left(1-\alpha^{\prime \prime}+\frac{1}{2} b^{2}+b \sqrt{2 \alpha^{\prime}}\right) P\right), \frac{1}{2} C\left(2 a^{2} P\left(1-\alpha^{\prime}-\alpha^{\prime \prime}\right)\right), \\
\left.\quad \frac{1}{2} C\left(\left(1+b^{2}+b \sqrt{2 \alpha^{\prime}}+b \sqrt{2 \alpha^{\prime \prime}}\right) P\right)\right\} .
\end{aligned}
$$

Proof: Follows from (9) directly by setting $\alpha_{1}^{\prime}=\alpha_{2}^{\prime}=$ $\alpha^{\prime}, \alpha_{1}^{\prime \prime}=\alpha_{2}^{\prime \prime}=\alpha^{\prime \prime}$, and $\alpha_{r}=1 / 2$.

Without backhaul, $X_{r}$ would only be partially known by the source nodes, i.e., $\alpha_{1}^{\prime \prime}=\alpha_{2}^{\prime \prime}=0$.

\section{Physical Layer Network Coding by Lattice Coding}

In contrast to Sec. III-A where $\mathcal{R}$ first decodes $\left(W_{1}, W_{2}\right)$ and then encodes into a joint $\mathrm{NC}$ message $W_{r}$, the relay can decode the NC message directly from $Y_{r}^{(n)}$ by using lattice encoding at the sources and lattice decoding at the relay, as in [8], [14] where only the case of symmetric powers is considered. We propose a protocol based on superposition of a lattice code and a random code to be able to handle the case of non-symmetric powers. Without loss of generality, we assume that $P_{1} \leq P_{2}$ (hence $R_{1} \leq R_{2}$ ). $\mathcal{S}_{2}$ splits its message $W_{2, t}$ into two parts $\left[W_{2, t}^{\prime}, W_{2, t}^{\prime \prime}\right]$, where $W_{2, t}^{\prime}$ has the same length as $W_{1, t} . \mathcal{S}_{1}$ encodes $W_{1, t}$ based on a nested lattice code [28], and we denote the corresponding transmitted codeword by $V_{1}^{(n)}\left(W_{1, t}\right)$. $\mathcal{S}_{2}$ encodes $W_{2, t}^{\prime}$ using the same nested lattice code as $\mathcal{S}_{1}$, denoting the corresponding codeword by $V_{2}^{(n)}\left(W_{2, t}^{\prime}\right)$, and encodes $W_{2, t}^{\prime \prime}$ using a random codebook $\left\{V_{3}^{(n)}\right\}$ of size $2^{n\left(R_{2}-R_{1}\right)}$. Note that codewords $V_{1}^{(n)}$ and $V_{2}^{(n)}$ are independent even though they are generated by the same nested lattice code, since the dither vectors used at $\mathcal{S}_{1}$ and $\mathcal{S}_{2}$ are independent [8], [28]. The relay, after decoding $W_{2, t-1}^{\prime \prime}$ via a single-user joint-typicality decoder and the NC message
$W_{1, t-1} \oplus W_{2, t-1}^{\prime}$ using a lattice decoder, encodes all these new messages by using an independent random codebook $\left\{U^{(n)}\right\}$ of size $2^{n R_{2}}$,

$$
X_{r, t}^{(n)}=\sqrt{P_{r}} U^{(n)}\left(W_{1, t-1} \oplus W_{2, t-1}^{\prime}, W_{2, t-1}^{\prime \prime}\right) .
$$

Since $W_{1, t-1}$ and $W_{2, t-1}$ are known both at $\mathcal{S}_{1}$ and $\mathcal{S}_{2}$ thanks to the backhaul, $U^{(n)}\left(W_{1, t-1} \oplus W_{2, t-1}^{\prime}, W_{2, t-1}^{\prime \prime}\right)$ is also known. Therefore $\mathcal{S}_{1}$ and $\mathcal{S}_{2}$ cooperate with $\mathcal{R}$ as follows

$$
\begin{aligned}
& X_{1, t}^{(n)}=\sqrt{\delta} V_{1}^{(n)}\left(W_{1, t}\right)+\sqrt{P_{1}-\delta} U^{(n)}, \\
& X_{2, t}^{(n)}=\sqrt{\delta} V_{2}^{(n)}\left(W_{2, t}^{\prime}\right)+\sqrt{\epsilon} V_{3}^{(n)}\left(W_{2, t}^{\prime \prime}\right)+\sqrt{P_{2}-\delta-\epsilon} U^{(n)},
\end{aligned}
$$

where $0 \leq \delta \leq P_{1}$ and $0 \leq \epsilon \leq P_{2}-\delta$ are the allocated power to transmit the new messages. The corresponding received signals at the relay and destinations are

$$
\begin{aligned}
Y_{r, t}^{(n)}= & a \sqrt{\delta}\left(V_{1}^{(n)}+V_{2}^{(n)}\right)+a \sqrt{\epsilon} V_{3}^{(n)} \\
& +a\left(\sqrt{P_{1}-\delta}+\sqrt{P_{2}-\delta-\epsilon}\right) U^{(n)}+Z_{r, t}^{(n)}, \\
Y_{1, t}^{(n)}= & \sqrt{\delta} V_{1}^{(n)}+\left(\sqrt{P_{1}-\delta}+b \sqrt{P_{r}}\right) U^{(n)}+Z_{1, t}^{(n)}, \quad \\
Y_{2, t}^{(n)}= & \sqrt{\delta} V_{2}^{(n)}+\sqrt{\epsilon} V_{3}^{(n)}+\left(\sqrt{P_{2}-\delta-\epsilon}+b \sqrt{P_{r}}\right) U^{(n)}+Z_{2, t}^{(n)} .
\end{aligned}
$$

$\mathcal{D}_{1}$ performs successive decoding: at the end of block $t$, $\mathcal{D}_{1}$ decodes $\left(W_{1, t-1} \oplus W_{2, t-1}^{\prime}, W_{2, t-1}^{\prime \prime}\right)$ from $Y_{1, t}^{(n)}$ by joint typicality and recovers $W_{2, t-1}^{\prime}$ by using $W_{1, t-1}$ which has been recovered successfully from block $t-1$; after cancelling out $U^{(n)}$ the new information $W_{1, t}$ can be decoded. This approach is also used for $\mathcal{D}_{2}$.

Proposition 3: Using lattice coding, an achievable rate region is given by

$$
\begin{aligned}
& R_{1}< \min \left\{C\left(-1 / 2+a^{2} \delta\right), C(\delta)\right\}, \\
& R_{2}<\min \left\{C\left(-1 / 2+a^{2} \delta+a^{2} \epsilon / 2\right), C(\delta+\epsilon)\right\}, \\
& R_{1}+R_{2}<\min \left\{C\left(P_{1}+b^{2} P_{r}+2 b \sqrt{P_{r}\left(P_{1}-\delta\right)}\right),\right. \\
&\left.C\left(P_{2}+b^{2} P_{r}+2 b \sqrt{P_{r}\left(P_{2}-\delta-\epsilon\right)}\right)\right\},
\end{aligned}
$$

with the union taken over $0 \leq \delta \leq P_{1}$ and $0 \leq \epsilon \leq P_{2}-\delta$.

Proof: The proof can be found in Appendix C.

The first term in $R_{1}\left(R_{2}\right)$ refers to the decoding constraint at $\mathcal{R}$ for the nested lattice code.

Corollary 3: For the symmetric scenario, the achievable rate region is

$$
\begin{array}{r}
R<\max _{0 \leq \alpha \leq 1} \min \left\{C(\alpha P), C\left(-1 / 2+a^{2} P \alpha\right),\right. \\
\left.\frac{1}{2} C\left(\left(1+b^{2}+2 b \sqrt{1-\alpha}\right) P\right)\right\} .
\end{array}
$$

Proof: The result follows straightforwardly from (14) by setting $\epsilon=0$ and $\delta=P \alpha$.

Without backhaul, the NC message would not be known at the sources, i.e., $\delta=P_{1}$ and $\epsilon=P_{2}-P_{1}$.

\section{Network Coding Based Beam-forming with DF $(D F+N B F)$}

To further exploit the available coherent combining (beamforming) gain [1]-[3] at the sinks, we propose a new strategy that performs $\mathrm{NC}$ at both $\mathcal{S}_{1}$ and $\mathcal{S}_{2}$ but not at the relay (decreasing the complexity at $\mathcal{R}$ ). We refer to this scheme as 
$\mathrm{NC}$ based beam-forming (NBF) since the signals transmitted at $\mathcal{S}_{1}, \mathcal{S}_{2}$ and $\mathcal{R}$ are formed in a beamforming-like fashion. NBF requires $B+2$ blocks in total: $\left(W_{1, t-1}, W_{2, t-1}\right)$ are exchanged via the backhaul during block $t-1$; at block $t$ the NC message $W_{t}=f\left(W_{1, t-1}, W_{2, t-1}\right)$ is transmitted; at block $t+1, W_{t}$ is transmitted by $\mathcal{R}$. The relay transmits $W_{t-1}$ using a random codebook $\left\{U^{(n)}\right\}$ of size $2^{n\left(R_{1}+R_{2}\right)}$. For each codeword $U^{(n)}\left(W_{t-1}\right)$, we generate an independent random codebook $\left\{V^{(n)}\right\}$ of size $2^{n\left(R_{1}+R_{2}\right)}$, and then use it to encode the new message $W_{t}$. We denote the selected codeword for $W_{t}$ given $W_{t-1}$ as $V^{(n)}\left(W_{t}, W_{t-1}\right)$. At block $t$, the transmitted signals are

$X_{r, t}^{(n)}=\sqrt{P_{r}} U^{(n)}\left(W_{t-1}\right)$,

$X_{1, t}^{(n)}=\sqrt{\alpha_{1} P_{1}} V^{(n)}\left(W_{t}, W_{t-1}\right)+\sqrt{\left(1-\alpha_{1}\right) P_{1}} U^{(n)}\left(W_{t-1}\right)$,

$X_{2, t}^{(n)}=\sqrt{\alpha_{2} P_{2}} V^{(n)}\left(W_{t}, W_{t-1}\right)+\sqrt{\left(1-\alpha_{2}\right) P_{2}} U^{(n)}\left(W_{t-1}\right)$,

where $0 \leq \alpha_{1}, \alpha_{2} \leq 1$ are power allocation parameters. The corresponding received signals are

$$
\begin{aligned}
Y_{1, t}^{(n)}= & \sqrt{\alpha_{1} P_{1}} V^{(n)}+\left(b \sqrt{P_{r}}+\sqrt{\left(1-\alpha_{1}\right) P_{1}}\right) U^{(n)}+Z_{1, t}^{(n)}, \\
Y_{2, t}^{(n)}= & \sqrt{\alpha_{2} P_{2}} V^{(n)}+\left(b \sqrt{P_{r}}+\sqrt{\left(1-\alpha_{2}\right) P_{2}}\right) U^{(n)}+Z_{2, t}^{(n)}, \\
Y_{r, t}^{(n)}= & a\left(\sqrt{\alpha_{1} P_{1}}+\sqrt{\alpha_{2} P_{2}}\right) V^{(n)} \\
& +a\left(\sqrt{\left(1-\alpha_{1}\right) P_{1}}+\sqrt{\left(1-\alpha_{2}\right) P_{2}}\right) U^{(n)}+Z_{r, t}^{(n)} .
\end{aligned}
$$

The decoding process is similar as in the other cooperative strategies: the relay performs successive decoding and the destinations utilize backward decoding.

Proposition 4: The achievable rate region for NBF is defined by

$$
\begin{array}{r}
R_{1}+R_{2}<\min \left\{C\left(P_{1}+b^{2} P_{r}+2 b \sqrt{\left(1-\alpha_{1}\right) P_{1} P_{r}}\right),\right. \\
C\left(P_{2}+b^{2} P_{r}+2 b \sqrt{\left(1-\alpha_{2}\right) P_{2} P_{r}}\right), \\
\left.C\left(a^{2}\left(\alpha_{1} P_{1}+\alpha_{2} P_{2}+2 \sqrt{\alpha_{1} \alpha_{2} P_{1} P_{2}}\right)\right)\right\},
\end{array}
$$

with the union taken over the power allocation parameters $0 \leq \alpha_{1}, \alpha_{2} \leq 1$.

Proof: Since $\mathcal{S}_{1}$ and $\mathcal{S}_{2}$ transmit the same NC message $W_{t}$, the achievable sum-rate can be split arbitrarily between them. Therefore in the NBF strategy only the constraints for the sum-rate matter. Following similar arguments as in Appendix A, the sum-rate constraint (55c) still holds here. By applying successive decoding to $Y_{r, t}^{(n)}$ and backward decoding to $Y_{1, t}^{(n)}$ and $Y_{2, t}^{(n)}$, the jointly Gaussian distributed random variables $\left(V^{(n)}, U^{(n)}\right)$ will translate (55c) into (17).

The terms in (17) indicate the constraints at $\mathcal{D}_{1}, \mathcal{D}_{2}$, and $\mathcal{R}$, respectively.

Corollary 4: For the symmetric scenario, the achievable rate region is defined by

$$
R<\max _{0 \leq \alpha \leq 1} \min \left\{\frac{1}{2} C\left(4 a^{2} P \alpha\right), \frac{1}{2} C\left(\left(1+b^{2}+2 b \sqrt{1-\alpha}\right) P\right)\right\} .
$$

Proof: The result follows straightforwardly from (17) by setting $\alpha_{1}=\alpha_{2}=\alpha$.

Without the backhaul, this strategy is impossible.

\section{Benchmark SCHEMES AND CUT-SET BOUnd}

To evaluate the performance of the cooperative NC strategies presented in Sec. III, we consider two benchmark schemes, namely the non-NC based time-sharing relay scheme and the non-DF based noisy NC scheme [15]. We also derive the cut-set bound [27] for our scenario.

\section{A. Time Sharing Relay with $D F(D F+T D)$}

In contrast to the orthogonal scheme described in [6] for the case of the IFRC, $\mathcal{S}_{1}$ and $\mathcal{S}_{2}$ here cooperate with $\mathcal{R}$ to convey both messages. We first generate two independent random codebooks $\left\{U_{1}^{(n)}\right\}$ of size $2^{n R_{1}}$ and $\left\{U_{2}^{(n)}\right\}$ of size $2^{n R_{2}}$, and they will be used by $\mathcal{R}$ to help $\mathcal{S}_{1}$ and $\mathcal{S}_{2}$, respectively. For each codeword in $\left\{U_{2}^{(n)}\right\}$, we generate an independent random codebook $\left\{V_{1}^{(n)}\right\}$ of size $2^{n R_{1}}$, and then use it to encode the new message at $\mathcal{S}_{1}$. Similarly we generate a random codebook $\left\{V_{2}^{(n)}\right\}$ of size $2^{n R_{2}}$ for each codeword in $\left\{U_{1}^{(n)}\right\}$. During block $t, W_{1, t}$ and $W_{2, t}$ are exchanged via the backhaul, and the transmission during block $t$ is divided into two parts. During the first part of block $t$, the transmitted signals are

$$
\begin{gathered}
X_{r, t_{1}}^{(n)}=\sqrt{P_{r}} U_{2}^{(n)}\left(W_{2, t-1}\right), \quad X_{2, t_{1}}^{(n)}=0, \\
X_{1, t_{1}}^{(n)}=\sqrt{\frac{\alpha_{1} P_{1}}{\beta}} V_{1}^{(n)}\left(W_{1, t}, W_{2, t-1}\right)+\sqrt{\frac{P_{1}\left(1-\alpha_{1}\right)}{\beta}} U_{2}^{(n)}\left(W_{2, t-1}\right),
\end{gathered}
$$

where $0 \leq \alpha_{1} \leq 1$ is the power allocation parameter and $0 \leq \beta \leq 1$ is the time sharing parameter. Transmission power $P_{1} / \beta$ is used in $X_{1, t_{1}}^{(n)}$ to meet the power constraint (2). The received signals are

$$
\begin{aligned}
& Y_{2, t_{1}}^{(n)}=b X_{r, t_{1}}^{(n)}+Z_{2, t_{1}}^{(n)}=b \sqrt{P_{r}} U_{2}^{(n)}+Z_{2, t_{1}}^{(n)}, \\
& Y_{r, t_{1}}^{(n)}=a \sqrt{\frac{\alpha_{1} P_{1}}{\beta}} V_{1}^{(n)}+a \sqrt{\frac{P_{1}\left(1-\alpha_{1}\right)}{\beta}} U_{2}^{(n)}+Z_{r, t_{1}}^{(n)}, \\
& Y_{1, t_{1}}^{(n)}=\sqrt{\frac{\alpha_{1} P_{1}}{\beta}} V_{1}^{(n)}+\left(\sqrt{\frac{P_{1}\left(1-\alpha_{1}\right)}{\beta}}+b \sqrt{P_{r}}\right) U_{2}^{(n)}+Z_{1, t_{1}}^{(n)} .
\end{aligned}
$$

The relay decodes $W_{1, t}$ given $W_{2, t-1}$ and then encodes it to $U_{1}^{(n)}\left(W_{1, t}\right)$. During the remaining part of block $t$, the transmitted signals are

$$
\begin{aligned}
& X_{r, t_{2}}^{(n)}=\sqrt{P_{r}} U_{1}^{(n)}\left(W_{1, t}\right), \quad X_{1, t_{2}}^{(n)}=0, \\
& X_{2, t_{2}}^{(n)}=\sqrt{\frac{\alpha_{2} P_{2}}{1-\beta}} V_{2}^{(n)}\left(W_{2, t}, W_{1, t}\right)+\sqrt{\frac{P_{2}\left(1-\alpha_{2}\right)}{1-\beta}} U_{1}^{(n)}\left(W_{1, t}\right) .
\end{aligned}
$$

The corresponding received signals are

$Y_{1, t_{2}}^{(n)}=b X_{r, t_{2}}^{(n)}+Z_{1, t_{2}}^{(n)}=b \sqrt{P_{r}} U_{1}^{(n)}+Z_{1, t_{2}}^{(n)}$,

$Y_{r, t_{2}}^{(n)}=a \sqrt{\frac{\alpha_{2} P_{2}}{1-\beta}} V_{2}^{(n)}+a \sqrt{\frac{P_{2}\left(1-\alpha_{2}\right)}{1-\beta}} U_{1}^{(n)}+Z_{2, t_{2}}^{(n)}$,

$Y_{2, t_{2}}^{(n)}=\sqrt{\frac{\alpha_{2} P_{2}}{1-\beta}} V_{2}^{(n)}+\left(\sqrt{\frac{\left(1-\alpha_{2}\right) P_{2}}{1-\beta}}+b \sqrt{P_{r}}\right) U_{1}^{(n)}+Z_{2, t_{2}}^{(n)}$.

At the end of block $t, \mathcal{R}$ decodes $W_{2, t}$ given $W_{1, t}$, and $\mathcal{D}_{1}$ can retrieve $\left(W_{1, t}, W_{2, t-1}\right)$ reliably using sliding-window decoding based on the received signals during block $t$. Similarly, after the first part of block $t+1, \mathcal{D}_{2}$ can decode $\left(W_{2, t}, W_{1, t}\right)$ reliably based on signals received from the first part of block $t+1$ and the second part of block $t$. 
Proposition 5: The achievable rate region for this time sharing strategy is defined by

$$
\begin{gathered}
R_{1}<\min \left\{\beta C\left(\frac{\alpha_{1} a^{2} P_{1}}{\beta}\right), \beta C\left(\frac{\alpha_{1} P_{1}}{\beta}\right)+(1-\beta) C\left(b^{2} P_{r}\right),\right. \\
\left.(1-\beta) C\left(b^{2} P_{r}+\frac{P_{2}}{1-\beta}+2 b \sqrt{\left(1-\alpha_{2}\right) P_{2} P_{r} /(1-\beta)}\right)\right\}, \\
R_{2}<\min \left\{(1-\beta) C\left(\frac{\alpha_{2} a^{2} P_{2}}{1-\beta}\right),(1-\beta) C\left(\frac{\alpha_{2} P_{2}}{1-\beta}\right)+\beta C\left(b^{2} P_{r}\right),\right. \\
\left.\quad \beta C\left(b^{2} P_{r}+\frac{P_{1}}{\beta}+2 b \sqrt{P_{1} P_{r}\left(1-\alpha_{1}\right) / \beta}\right)\right\}, \\
R_{1}+R_{2}<\min \{ \\
(1-\beta) C\left(b^{2} P_{r}\right)+\beta C\left(b^{2} P_{r}+\frac{P_{1}}{\beta}+2 b \sqrt{P_{1} P_{r}\left(1-\alpha_{1}\right) / \beta}\right), \\
\left.\beta C\left(b^{2} P_{r}\right)+(1-\beta) C\left(b^{2} P_{r}+\frac{P_{2}}{1-\beta}+2 b \sqrt{\frac{P_{2} P_{r}\left(1-\alpha_{2}\right)}{1-\beta}}\right)\right\},
\end{gathered}
$$

with the union taken over all $0 \leq \alpha_{1}, \alpha_{2} \leq 1$ and the time sharing parameter $0 \leq \beta \leq 1$.

Proof: The proof follows immediately from Appendix B by applying the Gaussian condition and noting the dependence stated in (19) and (20).

Constraints in $R_{1}\left(R_{2}\right)$ correspond to the condition of successful decoding of $W_{1}\left(W_{2}\right)$ at $\mathcal{R}, \mathcal{D}_{1}\left(\mathcal{D}_{2}\right)$, and $\mathcal{D}_{2}$ $\left(\mathcal{D}_{1}\right)$, respectively. Constraints in $R_{1}+R_{2}$ refer to successful decoding at $\mathcal{D}_{1}$ and $\mathcal{D}_{2}$.

By setting $\alpha_{1}=\alpha_{2}=\alpha$ and $\beta=1 / 2,(21)$ can be translated into the symmetric rate constraint

$$
\begin{aligned}
R< & \max _{0 \leq \alpha \leq 1} \min \left\{\frac{1}{2} C\left(P\left(2+b^{2}+2 b \sqrt{2-2 \alpha}\right)\right),\right. \\
& \frac{1}{2} C\left(\left(2 \alpha+b^{2}+2 \alpha b^{2} P\right) P\right), \frac{1}{2} C\left(2 a^{2} P \alpha\right), \\
& \left.\frac{1}{4}\left[C\left(b^{2} P\right)+C\left(\left(2+b^{2}+2 b \sqrt{2-2 \alpha}\right) P\right)\right]\right\} .
\end{aligned}
$$

Without backhaul, sources can only encode over their own messages. Therefore we have $\alpha_{1}=\alpha_{2}=1$ and the first term in (22) reduces to $\frac{1}{2} C\left(b^{2} P\right)$.

\section{B. Noisy Network Coding (Noisy NC)}

The basic principle of noisy NC, as described in [15], is to convey a "super message" $B$ times, each time using an independent codebook and letting $B \rightarrow \infty$, before the destination(s) can successfully decode the message. Therefore it is not clear how collaboration via the finite-rate backhaul can be implemented, since it requires a $B \rightarrow \infty$ times higher backhaul rate to exchange the super message before transmission starts. On the other hand, the backhaul provides orthogonal (i.e., out-ofband) conferencing bit-pipes between two source nodes. How to extend the noisy NC scheme [15], originally designed for relay networks with co-channel (i.e., in-band) transmission, so as to optimally utilize the rate-limited backhaul is interesting but out of the scope of this paper.

The achievable rate region for noisy NC (without backhaul collaboration) can be specialized from Theorem 1 of [15] to the multicast relay network in Fig. 1 as follows

$$
\begin{aligned}
R_{1}< & \min \left\{I\left(X_{1} ; \hat{Y}_{r} Y_{1} \mid X_{2} X_{r} Q\right), I\left(X_{1} ; \hat{Y}_{r} Y_{2} \mid X_{2} X_{r} Q\right),\right. \\
& I\left(X_{1} X_{r} ; Y_{1} \mid X_{2} Q\right)-I\left(Y_{r} ; \hat{Y}_{r} \mid X_{1} X_{2} X_{r} Y_{1} Q\right), \\
& \left.I\left(X_{1} X_{r} ; Y_{2} \mid X_{2} Q\right)-I\left(Y_{r} ; \hat{Y}_{r} \mid X_{1} X_{2} X_{r} Y_{2} Q\right)\right\}, \\
R_{2}< & \min \left\{I\left(X_{2} ; \hat{Y}_{r} Y_{1} \mid X_{1} X_{r} Q\right), I\left(X_{2} ; \hat{Y}_{r} Y_{2} \mid X_{1} X_{r} Q\right),\right. \\
& I\left(X_{2} X_{r} ; Y_{1} \mid X_{1} Q\right)-I\left(Y_{r} ; \hat{Y}_{r} \mid X_{1} X_{2} X_{r} Y_{1} Q\right),
\end{aligned}
$$

$$
\begin{gathered}
\left.I\left(X_{2} X_{r} ; Y_{2} \mid X_{1} Q\right)-I\left(Y_{r} ; \hat{Y}_{r} \mid X_{1} X_{2} X_{r} Y_{2} Q\right)\right\} \\
R_{1}+R_{2}<\min \left\{I\left(X_{1} X_{2} ; \hat{Y}_{r} Y_{1} \mid X_{r} Q\right), I\left(X_{1} X_{2} ; \hat{Y}_{r} Y_{2} \mid X_{r} Q\right),\right. \\
I\left(X_{1} X_{2} X_{r} ; Y_{1} \mid Q\right)-I\left(Y_{r} ; \hat{Y}_{r} \mid X_{1} X_{2} X_{r} Y_{1} Q\right) \\
\left.I\left(X_{1} X_{2} X_{r} ; Y_{2} \mid Q\right)-I\left(Y_{r} ; \hat{Y}_{r} \mid X_{1} X_{2} X_{r} Y_{2} Q\right)\right\}
\end{gathered}
$$

where $\hat{Y}_{r}$ is the compressed version of $Y_{r}, Q$ is the timesharing random variable, and the joint probability can be partitioned as $p(q) p\left(x_{1} \mid q\right) p\left(x_{2} \mid q\right) p\left(x_{r} \mid q\right) p\left(\hat{y}_{r} \mid x_{r}, y_{r}, q\right)$. By setting $Q=\emptyset$ and $\hat{Y}_{r}=Y_{r}+\hat{Z}$ with $\hat{Z} \sim N\left(0, \sigma^{2}\right)$, and applying (1) and (2), the achievable rate region in (23) is simplified to

$$
\begin{array}{r}
R_{1}<\min \left\{C\left(a^{2} P_{1} /\left(1+\sigma^{2}\right)\right), C\left(b^{2} P_{r}\right)-C\left(1 / \sigma^{2}\right)\right\}, \\
R_{2}<\min \left\{C\left(a^{2} P_{2} /\left(1+\sigma^{2}\right)\right), C\left(b^{2} P_{r}\right)-C\left(1 / \sigma^{2}\right)\right\}, \\
R_{1}+R_{2}<\min \left\{C\left(P_{1}+a^{2}\left(P_{1}+P_{2}+P_{1} P_{2}\right) /\left(1+\sigma^{2}\right)\right),\right. \\
C\left(P_{2}+a^{2}\left(P_{1}+P_{2}+P_{1} P_{2}\right) /\left(1+\sigma^{2}\right)\right), \\
\left.C\left(P_{1}+b^{2} P_{r}\right)-C\left(1 / \sigma^{2}\right), C\left(P_{2}+b^{2} P_{r}\right)-C\left(1 / \sigma^{2}\right)\right\},
\end{array}
$$

with the union taken over all $\sigma^{2}>0$. Note that redundant terms have been removed from $R_{1}$ and $R_{2}$.

For the symmetric scenario, the achievable rate region is

$$
\begin{aligned}
& R<\min \left\{C\left(\frac{a^{2} P}{1+\sigma^{2}}\right), \frac{1}{2} C\left(P+\frac{a^{2}\left(2 P+P^{2}\right)}{1+\sigma^{2}}\right),\right. \\
& \left.\quad C\left(b^{2} P\right)-C\left(1 / \sigma^{2}\right), \frac{1}{2} C\left(P+b^{2} P\right)-\frac{1}{2} C\left(1 / \sigma^{2}\right)\right\} .
\end{aligned}
$$

\section{Cut-Set Bound}

By the cut-set bound [27], the maximum achievable sum rate from the source nodes to any of the destinations can be no larger than the minimum of the mutual information flows across all possible cuts, maximized over a joint distribution for the transmitted signals. In our case, the cut-set bound between the two sources and each of the sink for the network in Fig. 1 can be derived based on four cuts, as demonstrated in Fig. 2, as follows (the dimension super script ${ }^{(n)}$ is suppressed to simplify the notation)

$$
\begin{aligned}
R_{1}+ & R_{2} \leq C_{\text {cut-set }}=\sup _{p\left(X_{1}, X_{2}, X_{r}\right)} \min \{ \\
& \frac{1}{n} I\left(X_{1} X_{2} ; Y_{1} Y_{r} \mid X_{r}\right), \frac{1}{n} I\left(X_{1} X_{2} X_{r} ; Y_{1}\right), \\
& \left.\frac{1}{n} I\left(X_{1} X_{2} ; Y_{2} Y_{r} \mid X_{r}\right), \frac{1}{n} I\left(X_{1} X_{2} X_{r} ; Y_{2}\right)\right\}+\epsilon_{n},
\end{aligned}
$$

where $\epsilon_{n} \rightarrow 0$ as $n \rightarrow \infty$, and $X_{1}, X_{2}$ and $X_{r}$ are potentially correlated.

As suggested in [29, chp. 17], to find the exact cut-set bound $C_{\text {cut-set }}$, we will first find an upper bound $C_{\text {upp }} \geq C_{\text {cut-set }}$ based on the technique used in [1], and then find a lower bound $C_{\text {cut-set, } \mathrm{G}} \leq C_{\text {cut-set }}$ by restricting the source distribution to Gaussian, and finally show that $C_{\text {cut-set, } \mathrm{G}}=C_{\text {upp }}$.

Following the conventional notation for the differential entropy $h(X)$ of a continuous valued random variable $X$, the mutual information corresponding to cut 2 can be written as

$$
\begin{gathered}
I\left(X_{1} X_{2} X_{r} ; Y_{1}\right)=h\left(Y_{1}\right)-h\left(Y_{1} \mid X_{1} X_{2} X_{r}\right) \\
\quad=h\left(Y_{1}\right)-h\left(Z_{1}\right)=h\left(Y_{1}\right)-\frac{n}{2} \log (2 \pi e) .
\end{gathered}
$$




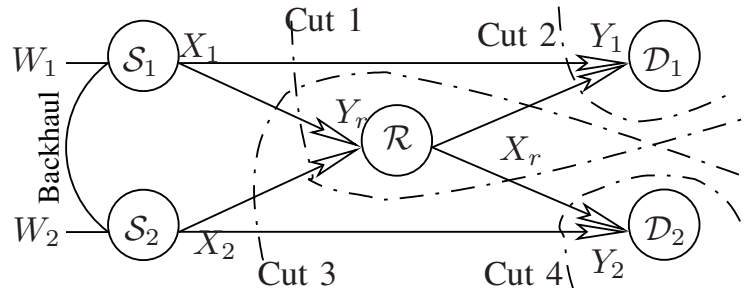

Figure 2. The sum multicast capacity is bounded by the cut-set bound based on the four cuts shown in the figure.

From the maximum entropy lemma [27], we get

$$
h\left(Y_{1}\right) \leq \sum_{i=1}^{n} h\left(Y_{1, i}\right) \leq \sum_{i=1}^{n} \frac{1}{2} \log \left(2 \pi e \operatorname{Var}\left[Y_{1, i}\right]\right),
$$

where the second equality is achieved when $Y_{1, i}$ is Gaussian distributed. Hence

$$
\begin{aligned}
\frac{1}{n} I\left(X_{1} X_{2} X_{r} ; Y_{1}\right) & \leq \frac{1}{n} \sum_{i=1}^{n} \frac{1}{2} \log \left(\operatorname{Var}\left(Y_{1, i}\right)\right) \\
& \leq \frac{1}{2} \log \left(\frac{1}{n} \sum_{i=1}^{n} \operatorname{Var}\left(Y_{1, i}\right)\right),
\end{aligned}
$$

where the last steps follow from Jensen's inequality. Furthermore, we have

$$
\begin{aligned}
\operatorname{Var}\left(Y_{1, i}\right) & =\operatorname{Var}\left(X_{1, i}+b X_{r, i}\right)+1 \leq 1+E\left[\left(X_{1, i}+b X_{r, i}\right)^{2}\right] \\
& =1+E\left[X_{1, i}^{2}\right]+b^{2} E\left[X_{r, i}^{2}\right]+2 b E\left[X_{1, i} X_{r, i}\right], \text { (30) }
\end{aligned}
$$

with equality for $E\left[X_{i}\right]=0$. Also, using the Cauchy-Schwarz inequality we get

$\frac{1}{n} \sum_{i=1}^{n} E\left[X_{1, i} X_{r, i}\right] \leq \sqrt{\frac{1}{n} \sum_{i=1}^{n} E\left[X_{r, i}^{2}\right] \frac{1}{n} \sum_{i=1}^{n} E\left[\left(E\left(X_{1, i} \mid X_{r, i}\right)\right)^{2}\right]}$.

As in [1], we can introduce an auxiliary variable $\alpha_{1} \in[0,1]$ such that

$$
\frac{1}{n} \sum_{i=1}^{n} E\left[\left(E\left(X_{1, i} \mid X_{r, i}\right)\right)^{2}\right]=\left(1-\alpha_{1}\right) P_{1} .
$$

Then by applying the power constraint defined in (2), we have

$$
\frac{1}{n} \sum_{i=1}^{n} E\left[\operatorname{Var}\left(X_{1, i} \mid X_{r, i}\right)\right]=\frac{1}{n} \sum_{i=1}^{n}\left(E\left[X_{1, i}^{2}\right]-E\left[\left(E\left(X_{1, i} \mid X_{r, i}\right)\right)^{2}\right]\right)
$$

$$
\leq \alpha_{1} P_{1} \text {. }
$$

Similarly, we can introduce $\alpha_{2} \in[0,1]$ such that

$$
\begin{gathered}
\frac{1}{n} \sum_{i=1}^{n} E\left[\left(E\left(X_{2, i} \mid X_{r, i}\right)\right)^{2}\right]=\left(1-\alpha_{2}\right) P_{2} \\
\frac{1}{n} \sum_{i=1}^{n} E\left[\operatorname{Var}\left(X_{2, i} \mid X_{r, i}\right)\right] \leq \alpha_{2} P_{2} .
\end{gathered}
$$

By substituting (32) into (31) we get

$$
\frac{1}{n} \sum_{i=1}^{n} E\left[X_{1, i} X_{r, i}\right] \leq \sqrt{\left(1-\alpha_{1}\right) P_{1} P_{r}}
$$

Now, substituting (36) and (30) into (29), and applying the same approach also to cut 4 , we get

$$
\begin{aligned}
& \frac{1}{n} I\left(X_{1} X_{2} X_{r} ; Y_{1}\right) \leq C\left(P_{1}+b^{2} P_{r}+2 b \sqrt{\left(1-\alpha_{1}\right) P_{1} P_{r}}\right), \\
& \frac{1}{n} I\left(X_{1} X_{2} X_{r} ; Y_{2}\right) \leq C\left(P_{2}+b^{2} P_{r}+2 b \sqrt{\left(1-\alpha_{2}\right) P_{2} P_{r}}\right) .
\end{aligned}
$$

For cut 1 we have

$$
\begin{aligned}
& I\left(X_{1} X_{2} ; Y_{1} Y_{r} \mid X_{r}\right)=h\left(Y_{1} Y_{r} \mid X_{r}\right)-h\left(Y_{1} Y_{r} \mid X_{1} X_{2} X_{r}\right) \\
& =h\left(Y_{1} Y_{r} \mid X_{r}\right)-h\left(Y_{1} \mid X_{1} X_{2} X_{r}\right)-h\left(Y_{r} \mid X_{1} X_{2} X_{r}\right) \\
& =h\left(Y_{1} Y_{r} \mid X_{r}\right)-h\left(Z_{1}\right)-h\left(Z_{r}\right)=h\left(Y_{1} Y_{r} \mid X_{r}\right)-n \log (2 \pi e) \\
& \leq \frac{1}{2} \sum_{i=1}^{n} \log \left((2 \pi e)^{2}\left|\boldsymbol{K}_{i}\right|\right)-n \log (2 \pi e)=\frac{1}{2} \sum_{i=1}^{n} \log \left(\left|\boldsymbol{K}_{i}\right|\right),
\end{aligned}
$$

where the second equality in (38) comes from the fact that $Y_{1}$ and $Y_{r}$ are independent given $\left(X_{1}, X_{2}, X_{r}\right)$ and the inequality is due to the maximum entropy lemma [27], with equality achieved by joint Gaussian distributed $\left(Y_{1, i}, Y_{r, i}\right)$ with conditional covariance matrices $\boldsymbol{K}_{i}$ defined by

$$
\boldsymbol{K}_{i}=\left[\begin{array}{cc}
E\left[\operatorname{Var}\left(Y_{1, i} \mid X_{r, i}\right)\right] & \left.E\left[\operatorname{Cov}\left(Y_{1, i}, Y_{r, i} \mid X_{r, i}\right)\right]\right], \\
E\left[\operatorname{Cov}\left(Y_{1, i}, Y_{r, i} \mid X_{r, i}\right)\right] & E\left[\operatorname{Var}\left(Y_{r, i} \mid X_{r, i}\right)\right]
\end{array}\right]
$$

where

$$
\begin{aligned}
& E\left[\operatorname{Var}\left(Y_{1, i} \mid X_{r, i}\right)\right]=1+E\left[\operatorname{Var}\left(X_{1, i} \mid X_{r, i}\right)\right], \\
& E\left[\operatorname{Cov}\left(Y_{1, i}, Y_{r, i} \mid X_{r, i}\right)\right]=a E\left[\operatorname{Cov}\left(X_{1, i}, X_{1, i}+X_{2, i} \mid X_{r, i}\right)\right] \\
& =a\left(E\left[\operatorname{Var}\left(X_{1, i} \mid X_{r, i}\right)\right]+E\left[\operatorname{Cov}\left(X_{1, i}, X_{2, i} \mid X_{r, i}\right)\right]\right), \\
& E\left[\operatorname{Var}\left(Y_{r, i} \mid X_{r, i}\right)\right]=1+a^{2} E\left[\operatorname{Var}\left(X_{1, i}+X_{2, i}\right) \mid X_{r, i}\right] \\
& =1+a^{2}\left(E\left[\operatorname{Var}\left(X_{1, i} \mid X_{r, i}\right)\right]+E\left[\operatorname{Var}\left(X_{2, i} \mid X_{r, i}\right)\right]\right. \\
& \left.+2 E\left[\operatorname{Cov}\left(X_{1, i}, X_{2, i} \mid X_{r, i}\right)\right]\right) .
\end{aligned}
$$

Obviously, the covariance matrices $\boldsymbol{K}_{i}$ are positive semidefinite. Since the function $\log |\boldsymbol{K}|$ is concave [30], we can thus bound the throughput of cut 1 as follows

$$
\begin{aligned}
\frac{1}{n} I\left(X_{1} X_{2} ; Y_{1} Y_{r} \mid X_{r}\right) & \leq \frac{1}{2} \sum_{i=1}^{n} \frac{1}{n} \log \left(\left|\boldsymbol{K}_{i}\right|\right) \\
& \leq \frac{1}{2} \log \left(\left|\frac{1}{n} \sum_{i=1}^{n} \boldsymbol{K}_{i}\right|\right) .
\end{aligned}
$$

It is then straightforward to show that for the inner term in (42) we can get

$$
\begin{gathered}
\left|\frac{1}{n} \sum_{i=1}^{n} \boldsymbol{K}_{i}\right|=1+\frac{1+a^{2}}{n} \sum_{i=1}^{n} E\left[\operatorname{Var}\left(X_{1, i} \mid X_{r, i}\right)\right] \\
+\frac{a^{2}}{n} \sum_{i=1}^{n} E\left[\operatorname{Var}\left(X_{2, i} \mid X_{r, i}\right)\right]+\frac{2 a^{2}}{n} \sum_{i=1}^{n} E\left[\operatorname{Cov}\left(X_{1, i}, X_{2, i} \mid X_{r, i}\right)\right] \\
+a^{2}\left(\frac{1}{n} \sum_{i=1}^{n} E\left[\operatorname{Var}\left(X_{1, i} \mid X_{r, i}\right)\right] \frac{1}{n} \sum_{i=1}^{n} E\left[\operatorname{Var}\left(X_{2, i} \mid X_{r, i}\right)\right]\right) \\
-a^{2}\left(\frac{1}{n} \sum_{i=1}^{n} E\left[\operatorname{Cov}\left(X_{1, i}, X_{2, i} \mid X_{r, i}\right)\right]\right)^{2}
\end{gathered}
$$


As $\operatorname{Cov}\left(X_{1, i}, X_{2, i} \mid X_{r, i}\right)=\phi_{i} \sqrt{\operatorname{Var}\left(X_{1, i} \mid X_{r, i}\right) \operatorname{Var}\left(X_{2, i} \mid X_{r, i}\right)}$, where $\left|\phi_{i}\right| \leq 1$ is the correlation coefficient, we have

$$
\begin{aligned}
& \frac{1}{n} \sum_{i=1}^{n} E\left[\operatorname{Cov}\left(X_{1, i}, X_{2, i} \mid X_{r, i}\right)\right] \\
& \leq \sqrt{\frac{1}{n} \sum_{i=1}^{n} \phi_{i} E\left[\operatorname{Var}\left(X_{1, i} \mid X_{r, i}\right)\right] \frac{1}{n} \sum_{i=1}^{n} \phi_{i} E\left[\operatorname{Var}\left(X_{2, i} \mid X_{r, i}\right)\right]} \\
& \leq \sqrt{\alpha_{1} \alpha_{2} P_{1} P_{2}}
\end{aligned}
$$

where the first inequality is due to the Cauchy-Schwarz inequality and the last step is given by (33) and (35). Given that $\left|\phi_{i}\right| \leq 1$, we can introduce another auxiliary variable $0 \leq \rho \leq 1$ such that

$$
\frac{1}{n} \sum_{i=1}^{n} E\left[\operatorname{Cov}\left(X_{1, i}, X_{2, i} \mid X_{r, i}\right)\right]=\rho \sqrt{\alpha_{1} \alpha_{2} P_{1} P_{2}} .
$$

Now, by substituting (33), (35) and (45) into (43), the bound (42) can be translated into

$$
\begin{aligned}
& \frac{1}{n} I\left(X_{1} X_{2} ; Y_{1} Y_{r} \mid X_{r}\right) \leq C\left(\alpha_{1} P_{1}\right. \\
& \left.\quad+a^{2}\left(\alpha_{1} P_{1}+\alpha_{2} P_{2}+2 \rho \sqrt{\alpha_{1} \alpha_{2} P_{1} P_{2}}+\left(1-\rho^{2}\right) \alpha_{1} \alpha_{2} P_{1} P_{2}\right)\right) .
\end{aligned}
$$

Similarly, we can bound the throughput of cut 3 as follows

$$
\begin{aligned}
& \frac{1}{n} I\left(X_{1} X_{2} ; Y_{2} Y_{r} \mid X_{r}\right) \leq C\left(\alpha_{2} P_{2}\right. \\
& \left.\quad+a^{2}\left(\alpha_{2} P_{2}+\alpha_{1} P_{1}+2 \rho \sqrt{\alpha_{1} \alpha_{2} P_{1} P_{2}}+\left(1-\rho^{2}\right) \alpha_{1} \alpha_{2} P_{1} P_{2}\right)\right) .
\end{aligned}
$$

By combining the individual bounds defined by (37), (46) and (47), and let $n \rightarrow \infty$, the cut-set bound $C_{\text {cut-set }}$ in (26) can be upper bounded by $C_{\text {upp }}$, as defined in (48) at the bottom of this page.

On the other hand, we can also lower bound $C_{\text {cut-set }}$ by $C_{\text {cut-set, },}$, obtained by restricting $p\left(X_{1}, X_{2}, X_{r}\right)$ in (26) to be Gaussian. We partition the Gaussian variables $X_{1}, X_{2}$ and $X_{r}$ as follows

$$
\begin{aligned}
& X_{r}=\sqrt{P_{r}} U, \\
& X_{1}=\sqrt{(1-\rho) \alpha_{1} P_{1}} S_{1}+\sqrt{\rho \alpha_{1} P_{1}} V+\sqrt{\left(1-\alpha_{1}\right) P_{1}} U, \\
& X_{2}=\sqrt{(1-\rho) \alpha_{2} P_{2}} S_{2}+\sqrt{\rho \alpha_{2} P_{2}} V+\sqrt{\left(1-\alpha_{2}\right) P_{2}} U,
\end{aligned}
$$

where $S_{1}, S_{2}, V$, and $U$ are $n$-dimensional independent Gaussian random vectors with zero-mean and unit-variance. $0 \leq \alpha_{1}, \alpha_{2}, \rho \leq 1$ are auxiliary variables introduced to represent the potential correlation among $X_{1}, X_{2}$ and $X_{r}$ due to cooperation. The received signals are then

$$
\begin{aligned}
Y_{1}=\sqrt{(1-\rho) \alpha_{1} P_{1}} S_{1} & +\left(b \sqrt{P_{r}}+\sqrt{\left(1-\alpha_{1}\right) P_{1}}\right) U \\
& +\sqrt{\rho \alpha_{1} P_{1}} V+Z_{1} \\
Y_{2}=\sqrt{(1-\rho) \alpha_{2} P_{2}} S_{2} & +\left(b \sqrt{P_{r}}+\sqrt{\left(1-\alpha_{2}\right) P_{2}}\right) U \\
& +\sqrt{\rho \alpha_{2} P_{2}} V+Z_{2} \\
Y_{r}=a \sqrt{(1-\rho) \alpha_{1} P_{1}} S_{1} & +a\left(\sqrt{\left(1-\alpha_{1}\right) P_{1}}+\sqrt{\left(1-\alpha_{2}\right) P_{2}}\right) U \\
+a \sqrt{(1-\rho) \alpha_{2} P_{2}} S_{2}+a & \left(\sqrt{\rho \alpha_{1} P_{1}}+\sqrt{\rho \alpha_{2} P_{2}}\right) V+Z_{r} .
\end{aligned}
$$

By substituting (50) into (27) and (38), we can derive from (26)

$$
\begin{array}{r}
C_{\text {cut-set, } \mathrm{G}}=\sup _{0 \leq \alpha_{1}, \alpha_{2}, \rho \leq 1} \min \frac{1}{2 n} \sum_{i=1}^{n}\left\{\log \left(\operatorname{Var}\left(Y_{1, i}\right)\right),\right. \\
\left.\log \left(\operatorname{Var}\left(Y_{2, i}\right)\right), \log \left(\left|\boldsymbol{K}_{1, i}\right|\right), \log \left(\left|\boldsymbol{K}_{2, i}\right|\right)\right\}+\epsilon_{n},
\end{array}
$$

where $\epsilon_{n} \rightarrow 0$ as $n \rightarrow \infty$, and for $i=1, \ldots, n$ we have

$$
\begin{array}{r}
\operatorname{Var}\left(Y_{1, i}\right)=1+P_{1}+b^{2} P_{r}+2 b \sqrt{\left(1-\alpha_{1}\right) P_{1} P_{r}}, \\
\operatorname{Var}\left(Y_{2, i}\right)=1+P_{2}+b^{2} P_{r}+2 b \sqrt{\left(1-\alpha_{2}\right) P_{2} P_{r}}, \\
\left|\boldsymbol{K}_{1, i}\right|=1+\left(1+a^{2}\right) \alpha_{1} P_{1}+a^{2} \alpha_{2} P_{2}+2 a^{2} \rho \sqrt{\alpha_{1} \alpha_{2} P_{1} P_{2}} \\
+a^{2}\left(1-\rho^{2}\right) \alpha_{1} \alpha_{2} P_{1} P_{2}, \\
\left|\boldsymbol{K}_{2, i}\right|=1+\left(1+a^{2}\right) \alpha_{2} P_{2}+a^{2} \alpha_{2} P_{2}+2 a^{2} \rho \sqrt{\alpha_{1} \alpha_{2} P_{1} P_{2}} \\
+a^{2}\left(1-\rho^{2}\right) \alpha_{1} \alpha_{2} P_{1} P_{2} .
\end{array}
$$

By substituting (52) into (51) we get $C_{\text {cut-set, G }}$ which actually equals to $C_{\text {upp }}$ as defined in (48), i.e., $C_{\text {cut-set, G }}=C_{\text {upp }}$. Recall that $C_{\text {cut-set, G }} \leq C_{\text {cut-set }} \leq C_{\text {upp }}$, we can finally conclude that

$$
C_{\text {cut-set }}=C_{\text {upp }} \text {, }
$$

i.e., the capacity upper bound defined in (48) is actually the cut-set bound.

For the symmetric scenario where $P_{1}=P_{2}=P_{r}=P$, by setting $\alpha=\alpha_{1}=\alpha_{2}$, the cut-set bound defined in (48) can be translated to the following constraint

$$
\begin{aligned}
& R<\sup _{0 \leq \alpha, \rho \leq 1} \min \left\{\frac{1}{2} C\left(P\left(1+b^{2}+2 b \sqrt{1-\alpha}\right)\right),\right. \\
& \left.\frac{1}{2} C\left(P\left[\left(1+2 a^{2}\right) \alpha+2 a^{2} \rho \alpha+a^{2}\left(1-\rho^{2}\right) \alpha^{2} P\right]\right)\right\} .
\end{aligned}
$$

\section{Achievability of the Cut-Set Bound by DF+NBF}

Proposition 6: In the symmetric scenario where $P_{1}=P_{2}=$ $P_{r}=P$ and $R_{1}=R_{2}=R, \mathrm{DF}+\mathrm{NBF}$ can achieve the cutset bound, i.e. (18) and (53) are equivalent, if and only if $\left(a^{2}, b^{2}, P\right)$ satisfy

$$
\left\{\begin{array}{l}
4 a^{2}>\max \left\{2,1+b^{2}\right\} \\
0<P \leq \frac{8 a^{2}\left(2 a^{2}-1\right)}{2 a^{2}\left(1+b^{2}\right)-b^{2}+\sqrt{\left(4 a^{2}-b^{2}\right)\left(4 a^{2}-1\right) b^{2}}} .
\end{array}\right.
$$

$$
\begin{array}{r}
C_{\text {upp }}=\sup _{0 \leq \alpha_{1}, \alpha_{2}, \rho \leq 1} \min \left\{C\left(P_{1}+b^{2} P_{r}+2 b \sqrt{\left(1-\alpha_{1}\right) P_{1} P_{r}}\right), C\left(P_{2}+b^{2} P_{r}+2 b \sqrt{\left(1-\alpha_{2}\right) P_{2} P_{r}}\right),\right. \\
C\left(\left(1+a^{2}\right) \alpha_{1} P_{1}+a^{2} \alpha_{2} P_{2}+2 a^{2} \rho \sqrt{\alpha_{1} \alpha_{2} P_{1} P_{2}}+a^{2}\left(1-\rho^{2}\right) \alpha_{1} \alpha_{2} P_{1} P_{2}\right), \\
\left.C\left(\left(1+a^{2}\right) \alpha_{2} P_{2}+a^{2} \alpha_{1} P_{1}+2 a^{2} \rho \sqrt{\alpha_{1} \alpha_{2} P_{1} P_{2}}+a^{2}\left(1-\rho^{2}\right) \alpha_{1} \alpha_{2} P_{1} P_{2}\right)\right\} .
\end{array}
$$






Figure 3. Upper bound for the normalized transmit power constraint $P / \sigma^{2}$ given different source-relay and relay-destination channel gains. Contour plots of the upper bound are shown at the bottom.

Proof: The proof can be found in Appendix D.

Proposition 6 states that there exists a large set of different source-relay channel gains and relay-destination channel gains, where the cut-set bound can be achieved by the DF+NBF strategy if the normalized $\left(\sigma^{2}=1\right)$ transmit power constraint $P$ is no larger than an upper bound defined in (54), as shown in Fig. 3. Therefore we can claim that even for non-degraded Gaussian relay channels, the capacity region for the system defined in Fig. 1 can be known for the scenarios defined by Proposition 6.

An intuitive interpretation of Proposition 6 is that (54) ensures the successful decoding at the relay node $\mathcal{R}$. In this scenario, the NBF achievable rate (18) and the cut-set bound (53) have the same active constraint on the MAC at $\mathcal{D}_{1}$ and $\mathcal{D}_{2}$, and therefore leads to tight capacity bounds. The upper bound on $P$ in (54) is to make sure that, given $a^{2}$ and $\alpha$, the second term (the constraint at $\mathcal{R}$ ) in cut-set bound (53) cannot be increased by reducing $\rho$ (otherwise we can increase (53) simply by decreasing $\alpha$ and $\rho$ ).

\section{More General Networks}

With a high-rate backhaul, the extension to non-symmetric channel gains is straightforward: Replacing $a, b$ with $a_{1}, a_{2}, b_{1}, b_{2}$ in the previous analysis where appropriate, we will get the achievable rate regions and the cut-set bound in the general case. However, the results for the symmetric scenario where $R_{1}=R_{2}=R$ has to be modified since setting $\alpha_{1}=\alpha_{2}$ may no longer be the optimal solution.

For a low-rate backhaul with capacity of $C_{0}$, i.e., only partial cooperation among the sources is possible, cooperative NC strategies can be formulated in the following way. By exploiting rate-splitting [31], we first partition each source message into two parts

$$
W_{1}=\left[W_{1 c}, W_{1 p}\right], \quad W_{2}=\left[W_{2 c}, W_{2 p}\right],
$$

and then divide all the four messages evenly into $B$ blocks $W_{1 c, t}, W_{1 p, t}, W_{2 c, t}, W_{2 p, t}$, each with $n R_{1 c}, n R_{1 p}$, $n R_{2 c}, n R_{2 p}$ bits, respectively. The sources then exchange $\left(W_{1 c, t}, W_{2 c, t}\right)$ over backhaul at rate $R_{1, c}+R_{2, c}<C_{0}$ to enable cooperative transmission. Therefore cooperative NC strategies



Figure 4. Achievable rate regions with transmitting power $P_{1} / \sigma^{2}=10 \mathrm{~dB}$, $P_{2} / \sigma^{2}=5 \mathrm{~dB}, P_{r} / \sigma^{2}=5 \mathrm{~dB}$, source-relay channel gain $a^{2}=5 \mathrm{~dB}$, and relay-destination channel gain $b^{2}=5 \mathrm{~dB}$. The cut-set outer bound is also plotted for reference. Curves for FNC and Lattice coding coincide each other.

presented in Sec. III are used to transmit $\left(W_{1 c, t}, W_{2 c, t}\right)$ and non-cooperative strategies are used to transmit $\left(W_{1 p, t}, W_{2 p, t}\right)$. Corresponding power allocation parameters need to be optimized.

\section{NumericAl Results}

In this section we present the achievable rate regions and the achievable equal rates $R$ for FNC, LNC, Lattice code, and NBF strategies, and compare them to two benchmark schemes and the cut-set bound.

\section{A. Achievable Rate Regions}

In Fig. 4, we plot the achievable rate regions for a scenario where source $\mathcal{S}_{1}$ has transmit power $P_{1} / \sigma^{2}=10 \mathrm{~dB}, \mathcal{S}_{2}$ has a power budget $P_{2} / \sigma^{2}=5 \mathrm{~dB}, \mathcal{R}$ has transmit power constraint $P_{r} / \sigma^{2}=5 \mathrm{~dB}$, the source-relay channel gain $a^{2}=5 \mathrm{~dB}$, and the relay-destination channel gain $b^{2}=5 \mathrm{~dB}$. Not surprisingly, the NBF scheme achieves the cut-set bound for this low to medium SNR region, which has been proved in Sec. IV-D for the symmetric scenarios. The curves for FNC and Lattice code coincide each other.

\section{B. Symmetric Achievable Rates}

In Fig. 5, we investigate the impact of the relay-destination link quality $b^{2}$ on the achievable rates for different cooperative strategies, with fixed transmit power $P / \sigma^{2}=5 \mathrm{~dB}$ and sourcerelay channel gain $a^{2}=10 \mathrm{~dB}$ and $a^{2}=5 \mathrm{~dB}$. With backhaul, substantial rate gains can be achieved by performing LNC or NBF compared to the time sharing relay. FNC or lattice coding is preferred for small $b^{2}$. As illustrated in the sub-figure, Significant gains can be achieved by utilizing the backhaul in the case of a poor relay-destination link (small $b^{2}$ ).

In Fig. 6 we fix $P / \sigma^{2}=5 \mathrm{~dB}$ and $b^{2}=0 \mathrm{~dB}$ instead and vary the source-relay link quality $a^{2}$. Rate gains of NC are significant in a large range of $a^{2}$ values. Note that when 

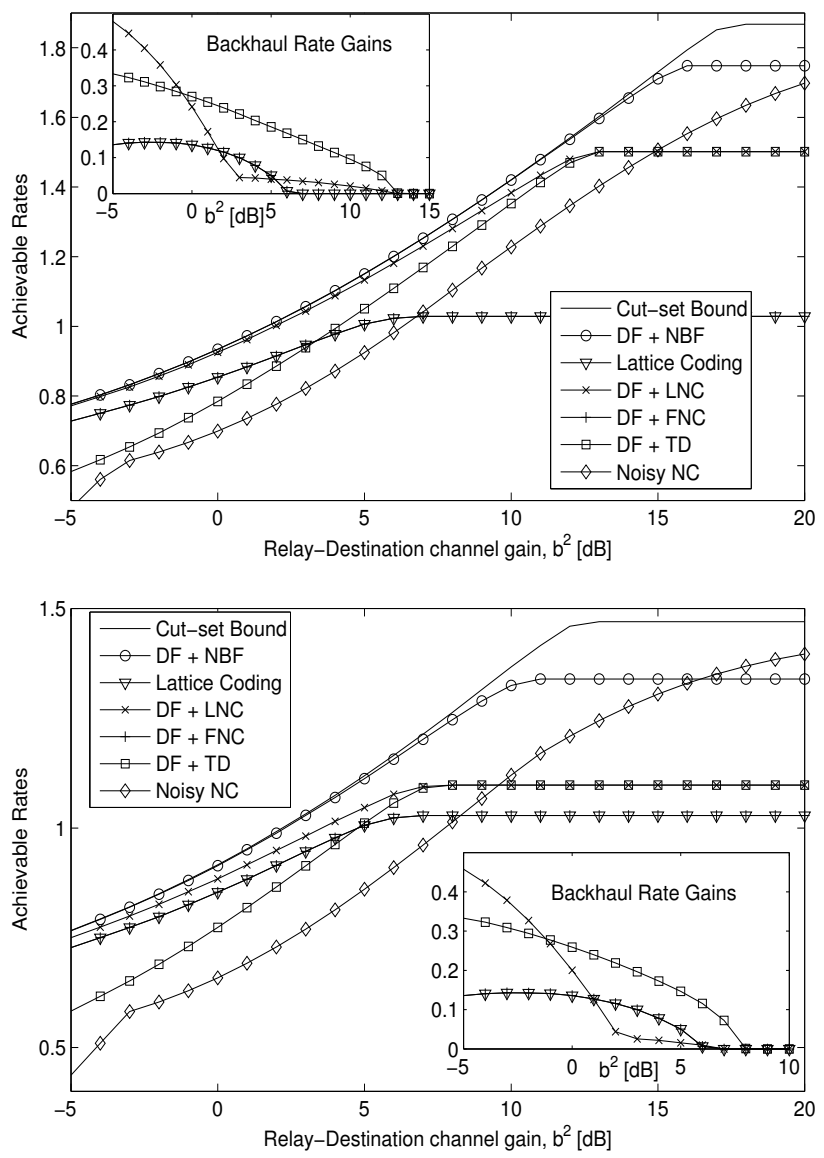

Figure 5. Effects of the relay-destination channel gain $b^{2}$ on the achievable rates with backhaul, when $P / \sigma^{2}=5 \mathrm{~dB}$ and the source-relay channel gain $a^{2}=10 \mathrm{~dB}$ (upper) and $5 \mathrm{~dB}$ (lower). The rate gains compared to the schemes without backhaul are also presented in the sub-figure.

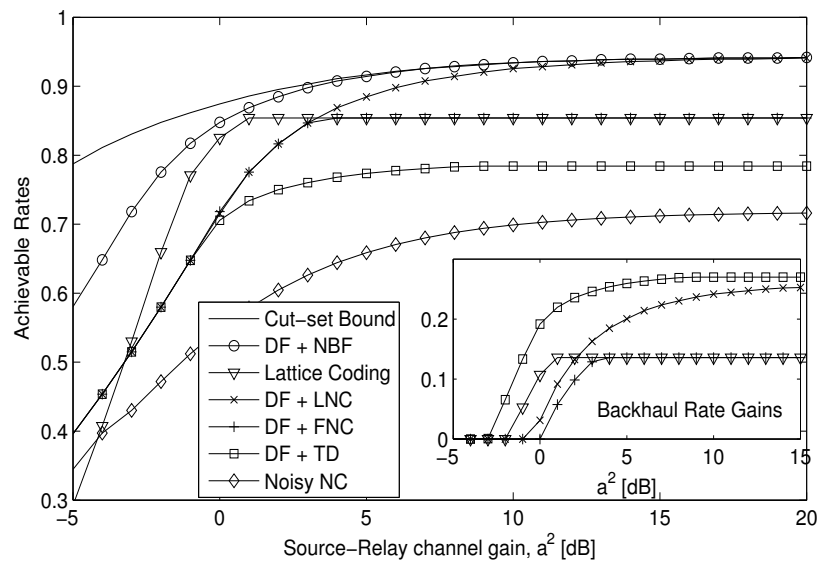

Figure 6. Effects of the source-relay channel gain $a^{2}$ on the achievable rates with $P / \sigma^{2}=5 \mathrm{~dB}$ and the relay-destination channel gain $b^{2}=0 \mathrm{~dB}$.

the source-relay link quality is comparable to the sourcedestination link, i.e. $a^{2}$ is around $0 \mathrm{~dB}$, the lattice coding strategy is preferred over LNC or FNC. The gain by using backhaul is significant for all schemes for $a^{2}$ larger than $0 \mathrm{~dB}$.

\section{Comparison of NBF with Lattice Coding}

To illustrate the performance of using lattice coding, we compared it to the NBF at fixed $P / \sigma^{2}=7 \mathrm{~dB}$, shown in Fig. 7 .
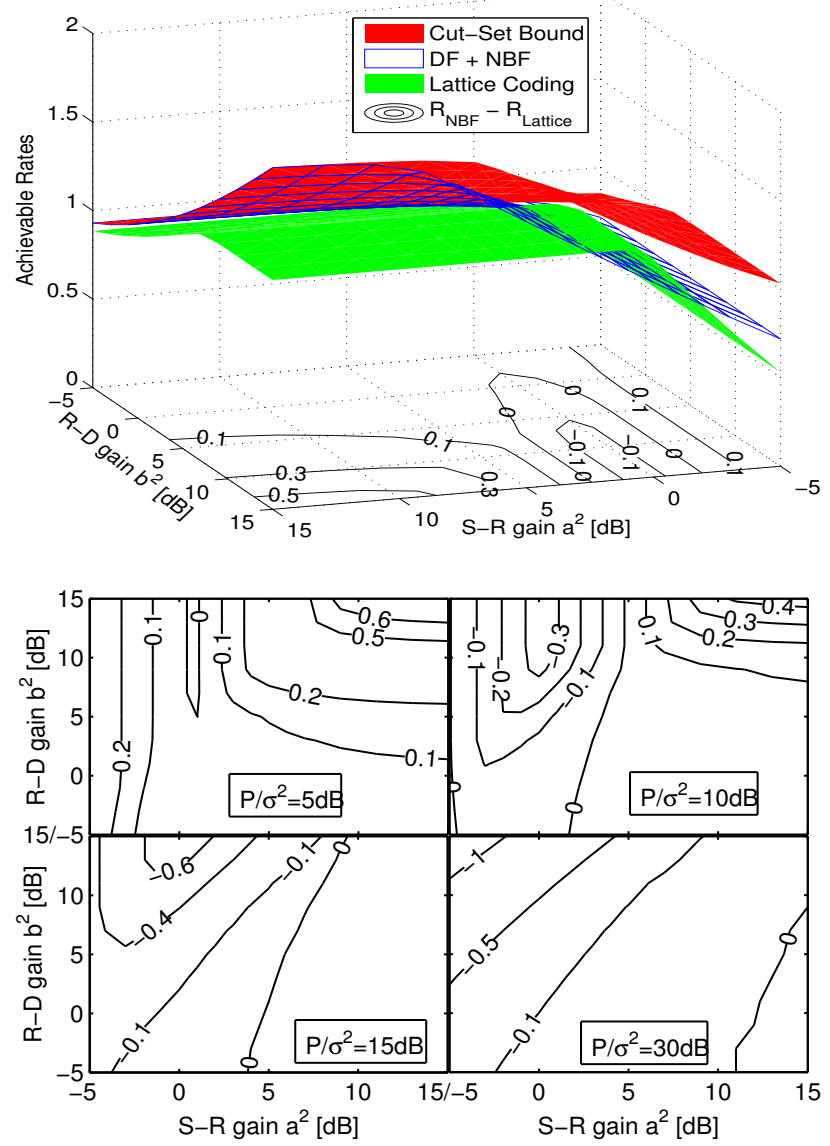

Figure 7. Comparison of DF+NBF (18) and Lattice Coding (14) with $P / \sigma^{2}=7 \mathrm{~dB}$ (upper). Contour plots given different transmit power constraints are also shown (lower).

The relative rate gain of NBF compared with lattice coding given different $P / \sigma^{2}$ is also shown as the contour plots. NBF outperforms lattice coding uniformly in low SNR regions $\left(P / \sigma^{2} \leq 5 \mathrm{~dB}\right)$ and in medium SNR regions $\left(5<P / \sigma^{2}<20 \mathrm{~dB}\right)$ with relatively strong source-relay gain $a^{2}$. For high SNR regions $\left(P / \sigma^{2}>20 \mathrm{~dB}\right)$, lattice coding outperforms NBF for most of channel conditions.

\section{CONCLUSIONS}

We have considered a relay-aided two-source two-sink wireless multicast network with a backhaul link between the source nodes. Different cooperative network coding strategies are investigated and compared with the cut-set bound and a benchmark strategy that does not use network coding, i.e., the relay is time shared by source nodes. Significant rate gains have been demonstrated. We have shown that the cutset bound can be achieved in certain channel configurations. In general, network coding based beam-forming (NBF) strategies give the best performance. In high SNR regions, however, the lattice code based strategy is preferred. FNC, which only performs modulo- 2 addition in the finite field, suffers limited performance loss in most of the cases. Further, and more importantly, we show significant rate gains compared to the scenarios without backhaul in various channel conditions. 


\section{APPENDIX A}

\section{PROOF OF PROPOSITION 1}

The achieved rate regions for MARC derived in [3], [4] involving multiple sources and a full-duplex DF relay can be directly applied here. Observing that $X_{r}^{(n)}$ is fully determined by $U^{(n)}$ as stated by (3) and that there is no cross links as defined by (1), the rate regions defined by (5) of [4] and (24), (25) of [3] can be translated to the FNC strategy as follows

$$
\begin{gathered}
n R_{1}<\min \left\{I\left(X_{1}^{(n)} ; Y_{r}^{(n)} \mid U^{(n)} X_{2}^{(n)}\right), I\left(X_{1}^{(n)} ; Y_{1}^{(n)} \mid U^{(n)}\right),\right. \\
\left.I\left(X_{r}^{(n)} ; Y_{2}^{(n)} \mid V_{2}^{(n)}\right)\right\}, \\
n R_{2}<\min \left\{I\left(X_{2}^{(n)} ; Y_{r}^{(n)} \mid U^{(n)} X_{1}^{(n)}\right), I\left(X_{2}^{(n)} ; Y_{2}^{(n)} \mid U^{(n)}\right),\right. \\
\left.I\left(X_{r}^{(n)} ; Y_{1}^{(n)} \mid V_{1}^{(n)}\right)\right\}, \\
n\left(R_{1}+R_{2}\right)<\min \left\{I\left(X_{1}^{(n)} X_{2}^{(n)} ; Y_{r}^{(n)} \mid U^{(n)}\right),\right. \\
\\
\left.\quad I\left(X_{1}^{(n)} X_{r}^{(n)} ; Y_{1}^{(n)}\right), I\left(X_{2}^{(n)} X_{r}^{(n)} ; Y_{2}^{(n)}\right)\right\},
\end{gathered}
$$

where $U, V_{1}, V_{2}$ are auxiliary random variables and the joint probability partitions as follows $p\left(V_{1}, V_{2}, U, X_{1}, X_{2}, X_{r}\right)=p\left(X_{1}, V_{1} \mid U\right) P\left(X_{2}, V_{2} \mid U\right) P\left(X_{r}, U\right)$. For constraints of $R_{1}$ in (55a), the first term corresponds to successful decoding of $W_{1}$ at $\mathcal{R}$ given that the relaying signal $X_{r}^{(n)}$ has been cancelled out and $\mathcal{S}_{2}$ is not transmitting. The second term refers to the decoding of $W_{1}$ at $\mathcal{D}_{1}$ given correctly decoded $W_{r}$. The last term indicates the successful decoding of $W_{r}$ (hence $W_{1}$ after NC decoding) at $\mathcal{D}_{2}$ given correctly decoded $W_{2}$. It is similar for $R_{2}$ and $R_{1}+R_{2}$.

Since $X_{1}-U-X_{2}$ forms a Markov chain, by following the similar arguments as in the proof of Lemma 3 in [18], one can show that there exist joint Gaussian variables $\left(X_{1}^{(n)}, X_{2}^{(n)}, U^{(n)}\right)$ such that the achievable rate region defined in (55) can be maximized. By choosing $U, V_{1}, V_{2}$ in (3) and (4) as i.i.d. zero-mean unit-variance random variables, we can see that $\left(X_{1}, X_{r}, X_{2}\right)$ is a zero-mean jointly Gaussian tuple satisfying the power constraint (2) and $X_{1}-X_{r}(U)-X_{2}$ forms a Markov chain. By substituting (5) into (55) and applying the Gaussian condition, one can get (6) straightforwardly.

\section{APPENDIX B}

PROOF OF PROPOSITION 2

By Theorem 1 of [6], $\mathcal{R}$ can decode $\left(W_{1, t}, W_{2, t}\right)$ reliably if $n$ is large, its past detection is correct, and

$$
\begin{array}{r}
n R_{1}<I\left(X_{1}^{(n)} ; Y_{r}^{(n)} \mid U_{1}^{(n)} U_{2}^{(n)} X_{2}^{(n)} X_{r}^{(n)}\right), \\
n R_{2}<I\left(X_{2}^{(n)} ; Y_{r}^{(n)} \mid U_{1}^{(n)} U_{2}^{(n)} X_{1}^{(n)} X_{r}^{(n)}\right), \\
n\left(R_{1}+R_{2}\right)<I\left(X_{1}^{(n)} X_{2}^{(n)} ; Y_{r}^{(n)} \mid U_{1}^{(n)} U_{2}^{(n)} X_{r}^{(n)}\right),
\end{array}
$$

where $U_{1}, U_{2}$ are auxiliary random variables and the joint probability partitions as follows

$p\left(U_{1}, U_{2}, X_{1}, X_{2}, X_{r}\right)=p\left(X_{1}, U_{1}\right) P\left(X_{2}, U_{2}\right) P\left(X_{r} \mid U_{1}, U_{2}\right)$.

For $i=1,2, \mathcal{D}_{i}$ can decode $W_{i, t-1}$ reliably if $n$ is large, its previously detection of $W_{i, t}$ is correct, and

$n R_{1}<\min \left\{I\left(X_{1}^{(n)} X_{r}^{(n)} ; Y_{1}^{(n)} \mid U_{2}^{(n)}\right), I\left(X_{r}^{(n)} ; Y_{2}^{(n)} \mid U_{2}^{(n)} X_{2}^{(n)}\right)\right\}$, $n R_{2}<\min \left\{I\left(X_{2}^{(n)} X_{r}^{(n)} ; Y_{2}^{(n)} \mid U_{1}^{(n)}\right), I\left(X_{r}^{(n)} ; Y_{1}^{(n)} \mid U_{1}^{(n)} X_{1}^{(n)}\right)\right\}$, $n\left(R_{1}+R_{2}\right)<\min \left\{I\left(X_{1}^{(n)} X_{r}^{(n)} ; Y_{1}^{(n)}\right), I\left(X_{2}^{(n)} X_{r}^{(n)} ; Y_{2}^{(n)}\right)\right\}$.
By choosing $U_{1}, U_{2}, V_{1}, V_{2}$ i.i.d. zero-mean unit-variance random Gaussian and applying the Gaussian conditions in (56) and (57), we obtain the rate region as defined in (9).

\section{APPENDIX C \\ ProOF OF PROPOSITION 3}

After cancelling out $U^{(n)}$ from $Y_{r, t}^{(n)}$, the relay can reliably decode $W_{2, t}^{\prime \prime}$ if $R_{2}-R_{1}<\frac{1}{2} \log \left(1+\frac{a^{2} \epsilon}{1+2 a^{2} \delta}\right)$ (by using a Gaussian codebook). Then $\mathcal{R}$ can further cancels $V_{3}^{(n)}$ out and uses the remaining signal to decoded the $\mathrm{NC}$ message by using lattice decoding [14], [28] if $R_{1}<\frac{1}{2} \log \left(\frac{1}{2}+a^{2} \delta\right)$. Therefore decoding at $\mathcal{R}$ will generate the following constraints

$$
\begin{aligned}
& R_{1}<C\left(-1 / 2+a^{2} \delta\right), \\
& R_{2}<\frac{1}{2} \log \left(\left(\frac{1}{2}+a^{2} \delta\right)\left(1+\frac{a^{2} \epsilon}{1+2 a^{2} \delta}\right)\right)=C\left(-\frac{1}{2}+a^{2} \delta+\frac{a^{2} \epsilon}{2}\right) .
\end{aligned}
$$

$\mathcal{D}_{1}$ and $\mathcal{D}_{2}$ can successfully decode $W_{1, t}$ and $W_{2, t}=$ $\left[W_{2, t}^{\prime}, W_{2, t}^{\prime \prime}\right]$, respectively, if

$$
R_{1}<\frac{1}{2} \log (1+\delta), R_{2}<\frac{1}{2} \log (1+\delta+\epsilon) .
$$

By using successive decoding at both $\mathcal{D}_{1}$ and $\mathcal{D}_{2}$, the following constraints apply

$$
\begin{aligned}
& R_{1}+R_{2}<\frac{1}{2} \log (1+\delta)+\frac{1}{2} \log \left(1+\frac{\left(\sqrt{P_{1}-\delta}+b \sqrt{P_{r}}\right)^{2}}{1+\delta}\right), \quad \\
& R_{1}+R_{2}<\frac{1}{2} \log (1+\delta+\epsilon)+\frac{1}{2} \log \left(1+\frac{\left(\sqrt{P_{2}-\delta-\epsilon}+b \sqrt{P_{r}}\right)^{2}}{1+\delta+\epsilon}\right) .
\end{aligned}
$$

Combine (58), (59) and (60) together we can obtain (13).

\section{APPENDIX D}

\section{ProOF OF PROPOSITION 6}

From (18) and (53) we can capture the effective power gain as follows

$$
\begin{gathered}
g_{\mathrm{NBF}}=\max _{0 \leq \alpha \leq 1} \min \left\{4 a^{2} \alpha, 1+b^{2}+2 b \sqrt{1-\alpha}\right\}, \\
g_{\text {cut-set }}=\sup _{0 \leq \alpha, \rho \leq 1} \min \left\{1+b^{2}+2 b \sqrt{1-\alpha},\right. \\
\\
\left.\alpha+a^{2}\left(2 \alpha+2 \alpha \rho+\left(1-\rho^{2}\right) \alpha^{2} P\right)\right\} .
\end{gathered}
$$

From (61) it is straightforward to shown that

$$
g_{\mathrm{NBF}}= \begin{cases}4 a^{2}, & \text { if } 4 a^{2} \leq 1+b^{2} ; \\ 1+b^{2}+2 b \sqrt{1-\alpha^{*}}, & \text { otherwise }\end{cases}
$$

where $a^{*} \in[0,1]$ satisfies $4 a^{2} \alpha^{*}=1+b^{2}+2 b \sqrt{1-\alpha^{*}}$. For the second part of (62), we have

$$
\begin{aligned}
& \max _{0 \leq \alpha, \rho \leq 1} \alpha+a^{2}\left(2 \alpha+2 \alpha \rho+\left(1-\rho^{2}\right) \alpha^{2} P\right) \\
= & \max _{0 \leq \alpha, \rho \leq 1} \alpha+a^{2}\left(2 \alpha+\alpha^{2} P+\frac{1}{P}-P(1 / P-\alpha \rho)^{2}\right) \\
= & \begin{cases}1+a^{2}\left(2+P+\frac{1}{P}\right), & \text { if } P>1, \quad \text { by setting } \alpha=1, \rho=\frac{1}{P} \text { ] } \\
1+4 a^{2}, & \text { if } P \leq 1, \quad \text { by setting } \alpha=\rho=1\end{cases}
\end{aligned}
$$

By combining (62) and (64) one can easily conclude that

$$
g_{\text {cut-set }}= \begin{cases}1+4 a^{2}, & \text { if } 4 a^{2} \leq b^{2} \text { and } P \leq 1 ; \\ >1+4 a^{2}, & \text { if } 4 a^{2} \leq b^{2} \text { and } P>1 ; \\ 1+b^{2}+2 b \sqrt{1-\alpha^{*}}, & \text { otherwise; }\end{cases}
$$

where $0 \leq \alpha^{*} \leq 1$ satisfies the equality

$1+b^{2}+2 b \sqrt{1-\alpha^{*}}=\alpha^{*}+a^{2}\left(2 \alpha^{*}+2 \alpha^{*} \rho+\left(1-\rho^{2}\right)\left(\alpha^{*}\right)^{2} P\right)$. 
From (65) it clearly follows that $g_{\text {cut-set }}>4 a^{2}$ for the scenarios when $4 a^{2} \leq 1+b^{2}$. Therefore $g_{\mathrm{NBF}}=g_{\text {cut-set }}$ is possible only if $4 a^{2}>1+b^{2}$, i.e., there should exist two variables $0 \leq \alpha^{*}, \rho \leq 1$ such that

$$
\begin{aligned}
& 4 a^{2} \alpha^{*}=1+b^{2}+2 b \sqrt{1-\alpha^{*}}, \\
& 4 a^{2} \alpha^{*}=\alpha^{*}+a^{2}\left(2 \alpha^{*}+2 \alpha^{*} \rho+\left(1-\rho^{2}\right)\left(\alpha^{*}\right)^{2} P\right) .
\end{aligned}
$$

By subtracting $1+b^{2}$ from both sides of (66a) and then taking square, we have

$$
16 a^{4}\left(\alpha^{*}\right)^{2}-\alpha^{*}\left(8 a^{2}+8 a^{2} b^{2}-4 b^{2}\right)+\left(1-b^{2}\right)^{2}=0,
$$

which has only one true root for (66a) (must satisfy $4 a^{2} \alpha^{*}>$ $\left.1+b^{2}\right)$

$$
\alpha^{*}=\frac{2 a^{2}\left(1+b^{2}\right)-b^{2}+\sqrt{\left(4 a^{2}-b^{2}\right)\left(4 a^{2}-a\right) b^{2}}}{8 a^{4}} .
$$

From (66b) we get

$$
\begin{aligned}
& \rho \alpha^{*}=1 / P+\sqrt{\alpha^{*} /\left(a^{2} P\right)+\left(\alpha^{*}-1 / P\right)^{2}}, \text { or } \\
& \rho \alpha^{*}=1 / P-\sqrt{\alpha^{*} /\left(a^{2} P\right)+\left(\alpha^{*}-1 / P\right)^{2}} .
\end{aligned}
$$

Since $0 \leq \rho \alpha^{*} \leq \alpha^{*}$, the first root is obviously a false root and therefore omitted. To make the second root satisfy the constraint, we must have

$$
0 \leq 1 / P-\sqrt{\alpha^{*} /\left(a^{2} P\right)+\left(\alpha^{*}-1 / P\right)^{2}} \leq \alpha^{*} .
$$

The second inequality is self-evident, and the first inequality requires

$$
a^{2}>1 / 2 \text { and } \alpha^{*} \leq \frac{1}{P}\left(2-\frac{1}{a^{2}}\right) .
$$

We therefore conclude from (67) and (68), given $4 a^{2}>1+b^{2}$ and $P>0$, that (66) holds if and only if $4 a^{2}>2$ and

$$
\frac{2 a^{2}\left(1+b^{2}\right)-b^{2}+\sqrt{\left(4 a^{2}-b^{2}\right)\left(4 a^{2}-a\right) b^{2}}}{8 a^{4}} \leq \frac{1}{P}\left(2-\frac{1}{a^{2}}\right) \text {. }
$$

Combined with the finding that $g_{\mathrm{NBF}}=g_{\text {cut-set }}$ is impossible for $4 a^{2} \leq 1+b^{2}$, we can conclude that $g_{\mathrm{NBF}}=g_{\text {cut-set }}$, i.e. (18) and (53) are identical, if and only if $\left(a^{2}, b^{2}, P\right)$ satisfies (54).

\section{ACKNOWLEDGMENTS}

The authors would like to thank the anonymous reviewers and the associate editor for their suggestions that helped improve the quality and presentation of the paper.

\section{REFERENCES}

[1] T. M. Cover and A. El Gamal, "Capacity theorems for the relay channel," IEEE Trans. Inf. Theory, vol. 25, pp. 572-584, Sep. 1979.

[2] A. Høst-Madsen and J. Zhang, "Capacity bounds and power allocation for wireless relay channels," IEEE Trans. Inf. Theory, vol. 51, pp. 2020 2040, Jun. 2005.

[3] G. Kramer, M. Gastpar and P. Gupta, "Cooperative strategies and capacity theorems for relay networks," IEEE Trans. Inf. Theory, vol. 51, pp. 30373063, Sep. 2005.

[4] G. Kramer and A. J. van Wijngaarden, "On the white Gaussian multipleaccess relay channel", in Proc. of IEEE ISIT, Jan. 2000.

[5] Y. Liang and G. Kramer, "Rate regions for relay broadcast channels," IEEE Trans. Inf. Theory, vol. 53, pp. 3517-3535, Oct. 2007.

[6] O. Sahin and E. Erkip, "Achievable rates for the Gaussian interference relay channel", in Proc. of IEEE GLOBECOM, Nov. 2007.

[7] D. Gündüz, O. Simeone, A. J. Goldsmith, H. V. Poor, and S. Shamai, "Relaying simultaneous multicast messages," in Proc. of IEEE ITW, Jun. 2009.
[8] D. Gündüz, O. Simeone, A. J. Goldsmith, H. V. Poor, and S. Shamai, "Multiple multicasts with the help of a relay," IEEE Trans. Inf. Theory, vol. 56, pp. 6142-6158, Dec. 2010.

[9] A. Avestimehr and T. Ho, "Approximate capacity of the symmetric halfduplex Gaussian butterfly network," in Proc. of IEEE ITW, Jun. 2009.

[10] S.R. Li, R.W. Yeung and N. Cai, "Linear Network Coding", IEEE Trans. Inf. Theory, vol. 49, No. 2, pp. 371-381, Feb. 2003.

[11] R. Koetter and M. Médard, "An algebraic approach to network coding", IEEE Trans. Networking, vol. 11, pp. 782-795, Oct. 2003.

[12] S. Katti, H. Rahul, W. Hu, D. Katabi, M. Médard, and J. Crowcroft, "XORs in the air: practical wireless network coding", IEEE/ACM Trans. Networking, vol. 16, pp. 497-510, Jun. 2008

[13] S. Katti, I. Marić, A. J. Goldsmith, D. Katabi, and M. Médard, "Joint relaying and network coding in wireless networks", in Proc. of IEEE ISIT, Jun. 2007.

[14] M. P. Wilson, K. Narayanan, H. D. Pfister, and A. Sprintson, "Joint physical layer coding and network coding for bi-directional relaying," IEEE Trans. Inf. Theory, vol. 56, No. 11, pp. 5641-5654, Nov. 2010.

[15] S. H. Lim, Y.-H. Kim, A. El Gamal and S.-Y. Chung, "Noisy network coding," IEEE Trans. Inf. Theory, vol. 57, No. 5, pp. 3132-3152, May 2011.

[16] F. M. J. Willems, "The discrete memoryless multiple-access channel with partially cooperating encoders," IEEE Trans. Inf. Theory, vol. 29, pp. 441-445, May 1983

[17] I. Marić, R. Yates, and G. Kramer, "Capacity of interference channels with partial transmitter cooperation," IEEE Trans. Inf. Theory, vol. 53, pp. 3536-3548, Oct. 2007.

[18] S. I. Bross, A. Lapidoth, and M. A. Wigger, "The Gaussian MAC with conferencing encoders," in Proc. of IEEE ISIT, Jul. 2008.

[19] N. Devroye, P. Mitran, and V. Tarokh, "Achievable rates in cognitive radio channels," IEEE Trans. Inf. Theory, vol. 52, pp. 1813-1827, May 2006.

[20] C. T. K Ng, N. Jindal, A. J. Goldsmith, and U. Mitra, "Capacity gain from two-transmitter and two-receiver cooperation", IEEE Trans. Inf. Theory, vol. 53, pp. 3822-3827, Oct. 2007.

[21] O. Simeone, D. Gündüz, H. V. Poor, A. J. Goldsmith, and S. Shamai, "Compound multiple-access channels with partial cooperation," IEEE Trans. Inf. Theory, vol. 55, pp. 2425-2441, Jun. 2009.

[22] J. N. Laneman, D. N. C. Tse, and G. W. Wornell, "Cooperative diversity in wireless networks: efficient protocols and outage behavior," IEEE Trans. Inf. Theory, vol. 50, pp. 3062-3080, Dec. 2004.

[23] B. Wang, J. Zhang, and A. Høst-Madsen, "On the capacity of MIMO relay channels," IEEE Trans. Inf. Theory, vol. 51, pp. 29-43, Jan. 2005.

[24] S. Simoens, O. Muñoz-Medina, J. Vidal, and A. del Coso, "On the Gaussian MIMO relay channel with full channel state information," IEEE Trans. Signal Proc., vol. 57, pp. 3588-3599, Sep. 2009.

[25] A. b. Carleial, "Multiple-access channels with different generalized feedback signals," IEEE Trans. Inf. Theory, vol. 28, pp. 841-850, Nov. 1982.

[26] F. M. J. Willems, Information Theoretical Results for the Discrete Memoryless Multiple Access Channel, Ph.D. thesis, Katholieke Univ. Leuven, Oct. 1982.

[27] T. M. Cover and J. A. Thomas, Elements of Information Theory, New York, Wiley, 2006.

[28] U. Erez and R. Zamir, "Achieving 1/2 log (1+ SNR) on the AWGN channel with lattice encoding and decoding," IEEE Trans. Inf. Theory, vol. 50, pp. 2293-2314, Oct. 2004.

[29] A. El Gamal and Y.-H. Kim, Lecture Notes on Network Information Theory, chp. 17, http://arxiv.org/abs/1001.3404

[30] T. M. Cover and J. A. Thomas, "Determinant inequalities via information theory," SIAM journal on Matrix Analysis and Applications, vol. 9, pp. 384-392, Jul. 1998.

[31] B. Rimoldi and R. Urbanke, "A rate-splitting approach to the Gaussian multiple-access channel," IEEE Trans. Inf. Theory, vol. 42, pp. 364-375, Mar. 1996. 


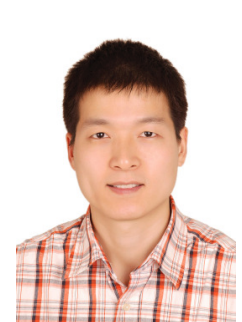

Jinfeng Du (S'07) received his B.Eng. degree in electronic information engineering from the University of Science and Technology of China (USTC), Hefei, China in 2004, his M.Sc. degree in electronic engineering, and his Tekn. Lic. degree in electronics and computer systems, both from the Royal Institute of Technology (KTH), Stockholm, Sweden, in 2006 and 2008, respectively. He is currently working for his $\mathrm{Ph} . \mathrm{D}$. degree in telecommunications at $\mathrm{KTH}$. $\mathrm{He}$ received the best paper award from IC-WCSP, Suzhou, October 2010, the "Hans Werthén Grant" from the Royal Swedish Academy of Engineering Science (IVA) in March 2011, and the grant from Ericsson's Research Foundation in May 2011.

Ming Xiao (S'2002-M'2007) was born in the

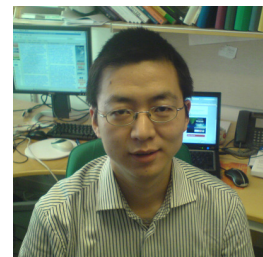

SiChuan Province, P. R. China, on May 22nd, 1975. He received Bachelor and Master degrees in Engineering from the University of Electronic Science and Technology of China, ChengDu in 1997 and 2002, respectively. He received Ph.D degree from Chalmers University of technology, Sweden in November 2007. From 1997 to 1999 , he worked as a network and software assistant engineer in ChinaTelecom. From 2000 to 2002, he also held a position in the SiChuan communications administration. From November 2007 to now, he has been in ACCESS Linnaeus center, school of electrical engineering, Royal Institute of Technology, Sweden, where he is currently an assistant professor. He received "Chinese Government Award for Outstanding Self-Financed Students Studying Aborad" in 2007. He got "Hans Werthén Grant" from royal Swedish academy of engineering science (IVA) in March 2006, and "Ericsson's research foundation" in 2010.



Mikael Skoglund (S'93-M'97-SM'04) received the Ph.D. degree in 1997 from Chalmers University of Technology, Sweden. In 1997, he joined the Royal Institute of Technology (KTH), Stockholm, Sweden, where he was appointed to the Chair in Communication Theory in 2003. At KTH, he heads the Communication Theory Lab and he is the Assistant Dean for Electrical Engineering.

Dr. Skoglund's research interests are in the theoretical aspects of wireless communications. $\mathrm{He}$ has worked on problems in source-channel coding. coding and transmission for wireless communications, Shannon theory and statistical signal processing. He has authored some 220 scientific papers, including papers that have received awards, invited conference presentations, and papers ranking as highly cited according to the ISI Essential Science Indicators. He has also consulted for industry, and he holds six patents.

Dr. Skoglund has served on numerous technical program committees for IEEE conferences. During 2003-08 he was an associate editor with the IEEE Transactions on Communications and he is presently on the editorial board for IEEE Transactions on Information Theory. 